\title{
Phenomenology of NNLO jet production at the LHC and its impact on parton distributions
}

\author{
Rabah Abdul Khalek ${ }^{1,2}$, Stefano Forte ${ }^{3}$, Thomas Gehrmann ${ }^{4}$, Aude Gehrmann-De Ridder ${ }^{4,5}$, Tommaso Giani ${ }^{6}$, \\ Nigel Glover $^{7}$, Alexander Huss ${ }^{7,8}$, Emanuele R. Nocera ${ }^{2}$, Joao Pires ${ }^{9}$, Juan Rojo ${ }^{1,2, a}$, Giovanni Stagnitto ${ }^{3,10,11}$ \\ ${ }^{1}$ Department of Physics and Astronomy, VU Amsterdam, 1081 HV Amsterdam, The Netherlands \\ ${ }^{2}$ Nikhef Theory Group, Science Park 105, 1098 XG Amsterdam, The Netherlands \\ 3 Tif Lab, Dipartimento di Fisica, Università di Milano and INFN, Sezione di Milano, Via Celoria 16, 20133 Milan, Italy \\ ${ }^{4}$ Department of Physics, University of Zurich, 8057 Zurich, Switzerland \\ ${ }^{5}$ Institute for Theoretical Physics, ETH, 8093 Zurich, Switzerland \\ ${ }^{6}$ The Higgs Centre for Theoretical Physics, University of Edinburgh, JCMB, KB, Mayfield Rd, Edinburgh EH9 3JZ, Scotland \\ ${ }^{7}$ Institute for Particle Physics Phenomenology, Durham University, Durham DH1 3LE, UK \\ ${ }^{8}$ Theoretical Physics Department, CERN, 1211, Geneva 23, Switzerland \\ ${ }^{9}$ LIP, Avenida Professor Gama Pinto 2, 1649-003 Lisboa, Portugal \\ ${ }^{10}$ Sorbonne Université, CNRS, Laboratoire de Physique Thórique et Hautes Énergies, 75005 Paris, France \\ ${ }^{11}$ Université Paris Diderot, 75013 Paris, France
}

Received: 27 May 2020 / Accepted: 4 August 2020 / Published online: 31 August 2020

(C) The Author(s) 2020

\begin{abstract}
We present a systematic investigation of jet production at hadron colliders from a phenomenological point of view, with the dual aim of providing a validation of theoretical calculations and guidance to future determinations of parton distributions (PDFs). We account for all available inclusive jet and dijet production measurements from ATLAS and CMS at 7 and $8 \mathrm{TeV}$ by including them in a global PDF determination, and comparing to theoretical predictions at NNLO QCD supplemented by electroweak (EW) corrections. We assess the compatibility of the PDFs, specifically the gluon, obtained before and after inclusion of the jet data. We compare the single-inclusive jet and dijet observables in terms of perturbative behaviour upon inclusion of QCD and EW corrections, impact on the PDFs, and global fit quality. In the single-inclusive case, we also investigate the role played by different scale choices and the stability of the results upon changes in modelling of the correlated experimental systematics.
\end{abstract}

\section{Contents}

1 Introduction . . . . . . . . . . . . . 1

2 Experimental data . . . . . . . . . . 2

2.1 Jet production at the LHC . . . . . . . . . 3

2.2 Jet data in NNPDF3.1 . . . . . . . . . . . 3

2.3 Jet data in this analysis $\ldots \ldots \ldots \ldots$

a e-mail: j.rojo@vu.nl (corresponding author)
3 Theoretical calculations and implementation .... 4

3.1 QCD corrections . . . . . . . . . 4

3.2 Electroweak corrections . . . . . . . . . . . 5

3.3 Implementation . . . . . . . . . . . 6

4 Results ................... . . 9

4.1 PDF sets . . . . . . . . . . . . . 9

4.2 Single-inclusive jets . . . . . . . . . . . . . 11

4.2.1 Impact and consistency of datasets . . . . 11

4.2.2 Impact of higher-order QCD and EW corrections ............ 13

4.2.3 Impact of the choice of scale . . . . . 15

4.2.4 Impact of the choice of correlation models 15

4.3 PDF fits with dijet data . . . . . . . . 17

4.3.1 Impact and consistency of datasets . . . 17

4.3.2 Impact of higher order QCD and EW corrections ............ 20

4.4 Single-inclusive jets vs. dijets: a comparative assessment . . . . . . . . . . . 20

5 Summary and outlook . . . . . . . . . . 22

References . . . . . . . . . . . . . 25

\section{Introduction}

The inclusive jet cross-section is the simplest hadron collider observable with a purely strongly interacting final state. The computation of next-to-next-to-leading order (NNLO) QCD corrections to it was completed recently [1-3] (see also 
Ref. [4]), and opens up the possibility of doing precision phenomenology with jet observables. Whereas single-inclusive jets have been used for the determination of the parton distributions (PDFs) of the proton [5] for over 30 years [6], there is a number of unsettled theoretical issues related to the definition of the observable which is most promising and appropriate for precision QCD studies, such as the determination of the PDFs and of the strong coupling constant $\alpha_{s}$.

The simplest inclusive observable, the single-inclusive jet cross-section $[7,8]$, has the undesirable feature of being nonunitary: each event is counted more than once, so the integral of the differential cross-section is not equal to the total cross-section. The dijet cross-section is free of this issue and it appears to be especially well-suited for PDF determination [9]. However, for this observable several scale choices are possible, because the more complex nature of the final state offers a wide choice of dimensionful kinematic variables; consequently, the significant scale dependence of NLO results has so far effectively prevented the use of this observable for PDF determination.

The availability of NNLO calculations has opened up the possibility of settling these issues, though their full understanding has posed a theoretical challenge, with the singleinclusive jet and dijet observables presenting different features. On the one hand, the issue of scale choice for the dijet observable has been essentially settled by the NNLO computation, with the scale dependence being under control at NNLO and the dijet invariant mass $m_{j j}$ emerging as the preferred choice. On the other hand, the single-inclusive jet cross-section has shown a dependence on the choice of scale which is not significantly reduced from NLO to NNLO [10], so that the understanding of the perturbative behavior, the scale dependence [11], and even the appropriate definition [12] of this observable are non-trivial. A careful analysis reveals that the apparent lack of improvement of scale stability from NLO to NNLO is due to an accidental NLO scale cancellation which occurs for particular values of the jet radius $[12,13]$. The persistence of a dependence on the central scale choice at NNLO can in turn be understood as a consequence of infrared sensitivity, which is aggravated by particular scale choices [11]. It then appears that the nonunitary definition of the observable is in fact necessary for perturbative stability, with dijets offering essentially the only viable unitary stable alternative [12]. From these studies the partonic transverse energy $\widehat{H}_{T}$ emerges as the optimal scale choice [11] for the calculation of single-inclusive jet crosssections.

In this work, we address these issues from a phenomenological point of view, specifically within the context of a global PDF determination: we study the effect of adding jet cross-sections to a global dataset, with various choices of the observable (single-inclusive jet, or dijet) and of the scale. In each case, we assess the fit quality and the impact of the jet data on the PDFs, at various perturbative orders. This allows us to achieve two main goals. First, we can test phenomenologically the conclusions of past theoretical studies [1-3,1012], by checking which observable and which scale choice leads to better perturbative stability, better PDF compatibility with other data and better fit quality, and more stringent constraints on the PDFs. Secondly, these results make it possible to optimize the choice of jet observables in view of their inclusion in future global PDF fits, and assess their impact as a means of PDF determination.

We will consider the complete inclusive jet [14-17] and dijet $[16,18,19]$ dataset from ATLAS and CMS at $\sqrt{s}=7$ and $8 \mathrm{TeV}$. Whereas most recent global determinations of the proton PDFs [20-22] include some of these jet datasets (for instance, NNPDF3.1 included the ATLAS and CMS singleinclusive data with $\sqrt{s}=2.76$ and $7 \mathrm{TeV}$ ), and other studies have assessed the impact of some jet measurements on smaller datasets $[19,23]$, this is the first time that the full LHC-Run I jet dataset is being considered, and specifically the first time dijet data are included in a modern global PDF determination.

Thanks to the availability of such a wide dataset, we will be able to pursue the two main goals discussed above, by including these jet data in the NNPDF3.1 dataset, while keeping the rest of the global dataset and adopting the same general PDF fitting methodology. In addition, we will look into two further secondary issues. First, we will study the impact of the inclusion of electroweak (EW) corrections to jet predictions. Second, we will assess the sensitivity of results to the treatment of experimental correlated systematic uncertainties, thus addressing the issue, recently raised e.g. in Ref. [24], of the sensitivity of some LHC jet datasets to variations in the experimental correlation model, which may lead to substantial differences in fit quality.

The outline of this paper is as follows. In Sect. 2 we discuss the experimental data for single-inclusive jet and inclusive dijet production. In Sect. 3 we present the theory, and in particular discuss NNLO QCD and EW corrections and scale choices. Our results for the global PDF analyses that we performed are presented in Sect. 4, where we also discuss their implications and summarize our findings. Future implications and avenues for further research are briefly addressed in Sect. 5.

\section{Experimental data}

We now discuss the single-inclusive jet and dijet data. We first summarize available inclusive jet production data from the LHC. We then review the jet cross-sections included in the NNPDF3.1 PDF determination and their treatment. We finally provide details on the treatment and kinematic cover- 
age of the single-inclusive jet and dijet datasets that we will use in this paper.

\subsection{Jet production at the LHC}

The ATLAS and CMS collaborations have performed a number of measurements of the single-inclusive and dijet crosssections at different center of mass energies, ranging from $\sqrt{s}=2.76$ to $13 \mathrm{TeV}$. In this work, we will focus on the 7 and $8 \mathrm{TeV}$ data, for which single-inclusive and dijet data corresponding to the same underlying dataset and integrated luminosity can be compared.

The $\sqrt{s}=7$ and $8 \mathrm{TeV}$ data are summarized in Table 1 , where for each dataset we indicate the experiment, the measured quantity, the center of mass energy $\sqrt{s}$, the integrated luminosity $\mathcal{L}$, the number of datapoints $n_{\text {dat }}$, and the published reference. All measurements are performed using the anti- $k_{t}$ algorithm [25] in the four-momentum recombination scheme, which leads to jets with non-vanishing invariant mass.

The relevant kinematic variables are defined as follows.

For single-inclusive jets, $p_{T}$ and $y$ are the jet transverse momentum and rapidity.

For dijets, $m_{j j}$ is the dijet invariant mass, $y^{*}=\left|y_{1}-y_{2}\right| / 2$ and $\left|y_{\max }\right|=\max \left(\left|y_{1}\right|,\left|y_{2}\right|\right)$ are respectively the absolute rapidity difference and maximum absolute rapidity of the two leading jets of the event.

Finally, for dijet triple-differential distributions, $p_{T, \text { avg }}=$ $\left(p_{T, 1}+p_{T, 2}\right) / 2$ is the average transverse momentum of the two leading jets, and $y_{b}=\left|y_{1}+y_{2}\right| / 2$ is the boost of the dijet system.

In addition to the data listed in Table 1, ATLAS and CMS have also performed measurements at $\sqrt{s}=13 \mathrm{TeV}$, though so far with smaller integrated luminosities than for their Run I counterparts: at Run II, the single-inclusive jet measurements from ATLAS [26] and CMS [27] have $\mathcal{L}=3.2 \mathrm{fb}^{-1}$ and $\mathcal{L}=71 \mathrm{pb}^{-1}$ respectively, while the dijet measurements from ATLAS [26] and CMS [28] have $\mathcal{L}=3.2 \mathrm{fb}^{-1}$ and $\mathcal{L}=2.3 \mathrm{fb}^{-1}$. For this reason, we do not include these datasets. Very recently, CMS has presented a single-inclusive jet measurement at $\sqrt{s}=13 \mathrm{TeV}$, based on a luminosity of $\mathcal{L}=35.9 \mathrm{fb}^{-1}[29]$.

We will also not include single-inclusive jet data at $\sqrt{\mathrm{s}}=$ 2.76 TeV [30,31] and 5.02 TeV [32]. The main motivation for these measurements was to provide a baseline for proton-lead and lead-lead data taken at the same center of mass energy. A possible exception could be the 5.02 TeV CMS doubledifferential cross-section data, based on an integrated luminosity of $\mathcal{L}=27.4 \mathrm{pb}^{-1}$ : indeed, a recent study [33] claims that they might also impact the proton PDFs. We will investigate this dataset in a follow-up study based on an update of the nNNPDF1.0 analysis [34] of nuclear parton distribution functions.
In addition, ATLAS and CMS have also presented several measurements of multijet ( $\geq 3$ jets) production. For example, ATLAS has provided measurements of three jet crosssections at $7 \mathrm{TeV}$ [35], differential in three-jet mass $m_{j j j}$ and the sum of the absolute rapidity separations between the three leading jets, $\left|y_{*}\right|$; and of four-jet cross-sections at $8 \mathrm{TeV}$ [36], differential in the $p_{T}$ of the four leading jets in the event.

CMS also has a measurement of the 3 -jet production crosssection at $7 \mathrm{TeV}$ [37] differential in the invariant mass of the three jets $m_{j j j}$. Because theoretical predictions are currently only available up to NLO for these observables, they will not be considered here, though they are important for other applications such as the validation of Monte Carlo event generators and searches for physics beyond the Standard Model.

\subsection{Jet data in NNPDF3.1}

The present study will be based on the PDF fitting framework adopted for the NNPDF3.1 global PDF determination [20]. As already mentioned, the NNPDF3.1 dataset includes several single-inclusive jet data. Specifically, for ATLAS the $\sqrt{s}=7 \mathrm{TeV}$ data from 2010 [38] and 2011 [14] and the $\sqrt{s}=2.76 \mathrm{TeV}$ [30] data (including cross-correlations between the $2.76 \mathrm{TeV}$ and the $7 \mathrm{TeV}$ data). For the $20117 \mathrm{TeV}$ data only the central rapidity bin $\left(y_{\text {jet }} \leq 0.5\right)$ was included, due to the difficulty in achieving a satisfactory description of the complete set of rapidity bins using the default experimental covariance matrix. From the CMS experiment, NNPDF3.1 included the measurements at $\sqrt{s}=7 \mathrm{TeV}$ [16] and 2.76 $\mathrm{TeV}$ [31], with their cross-correlations. Finally the CDF Run II data with the $k_{T}$ algorithm [39] was also included. Note that the value of the jet radius $R$ is different for each of these measurements: $R=0.4$ for the ATLAS 7 TeV 2010 and the $2.76 \mathrm{TeV}$ measurements; $R=0.6$ for the ATLAS $7 \mathrm{TeV}$ 2011 measurement; and $R=0.7$ for the CDF and CMS measurements.

In the default NNPDF3.1 PDF determination, theory predictions for all these data were obtained by combining NLO coefficient functions with NNLO perturbative evolution, because full NNLO results were not available then. In order to account for the missing NNLO corrections, a missing higher order uncertainty, estimated from scale variations, was added to jet data, as a fully correlated systematics. A variant PDF set was also produced by only including the two datasets for which the NNLO corrections were available at the time, namely the ATLAS and CMS 7 TeV 2011 data, with all the remaining jet data removed, and now using full NNLO theory. This reduced, but fully NNLO, dataset was also used for the determination of the strong coupling in Ref. [40], for the PDFs with QED corrections [41] and the PDFs with small- $x$ resummation [42], and for the recent studies of theoretical uncertainties on PDFs $[43,44]$. In all these previous studies, 
Table 1 The LHC single-inclusive jet and dijet cross-section data that will be used in this study. For each dataset we indicate the experiment, the measurement, the center of mass energy $\sqrt{s}$, the luminosity $\mathcal{L}$, the jet radius $R$, the measured distribution, the number of datapoints $n_{\text {dat }}$ and the reference

\begin{tabular}{llllllll}
\hline Experiment & Measurement & $\sqrt{s}[\mathrm{TeV}]$ & $\mathcal{L}\left[\mathrm{fb}^{-1}\right]$ & $R$ & Distribution & $n_{\text {dat }}$ & References \\
\hline ATLAS & Inclusive jets & 7 & 4.5 & 0.6 & $d^{2} \sigma / d p_{T} d|y|$ & 140 & {$[14]$} \\
CMS & Inclusive jets & 7 & 4.5 & 0.7 & $d^{2} \sigma / d p_{T} d|y|$ & 133 \\
ATLAS & Inclusive jets & 8 & 20.2 & 0.6 & $d^{2} \sigma / d p_{T} d|y|$ & {$[16]$} & 171 \\
CMS & Inclusive jets & 8 & 19.7 & 0.7 & $d^{2} \sigma / d p_{T} d|y|$ & {$[15]$} \\
ATLAS & Dijets & 7 & 4.5 & 0.6 & $d^{2} \sigma / d m_{j j} d\left|y^{*}\right|$ & 185 & {$[17]$} \\
CMS & Dijets & 7 & 4.5 & 0.7 & $d^{2} \sigma / d m_{j j} d\left|y_{\max }\right|$ & 54 & {$[18]$} \\
CMS & Dijets & 8 & 19.7 & 0.7 & $d^{3} \sigma / d p_{T, \text { avg }} d y_{b} d y^{*}$ & 122 & {$[19]$} \\
\end{tabular}

the renormalization and factorization scales were set equal to the jet transverse momentum, $\mu_{F}=\mu_{R}=p_{T \text {,jet }}$.

\subsection{Jet data in this analysis}

The single-inclusive jet data from ATLAS and CMS used in this work are the double-differential $\left(y, p_{T}\right)$ distributions listed in Table 1 . The ATLAS $7 \mathrm{TeV}$ data cover the range $100 \mathrm{GeV} \leq p_{T} \leq 1.992 \mathrm{TeV}$ and $0 \leq|y| \leq 3$, while the ATLAS $8 \mathrm{TeV}$ data cover the same rapidity range, but with an extended range of transverse momenta, namely $70 \mathrm{GeV} \leq$ $p_{T} \leq 2.5 \mathrm{TeV}$. In our default fit we include only the central rapidity bin $\left(y_{\text {jet }} \leq 0.5\right)$ of the ATLAS $7 \mathrm{TeV}$, for ease of comparison with NNPDF3.1. This is not expected to affect results, as in Ref. [20] it was shown that PDFs fitted to the central rapidity bin provide an equally good fit to all other rapidity bins, and in Ref. [45] it was checked explicitly that PDFs determined including each rapidity bin from this data in turn are indistinguishable. We will revisit this issue in Sect. 4.2.4, where we will discuss variant fits in which all rapidity bins are included, and we will consider alternative correlation models both for these data and for their $8 \mathrm{TeV}$ counterpart, as suggested in Refs. [15,24].

The CMS $7 \mathrm{TeV}$ data cover the range $100 \mathrm{GeV} \leq$ $p_{T} \leq 2.0 \mathrm{TeV}$ and $0 \leq|y| \leq 2.5$, and the CMS $8 \mathrm{TeV}$ data the extended range $74 \mathrm{GeV} \leq p_{T} \leq 2.5 \mathrm{TeV}$ and $0 \leq|y| \leq 3.0$. We note that in the case of the CMS $8 \mathrm{TeV}$ single-inclusive jets, measurements for $p_{T}<74 \mathrm{GeV}$ are also available, but these are excluded from the fit because non-perturbative and resummation corrections, not accounted for by fixed-order computations, are large at small $p_{T}$. We therefore retain only 185 points out of a total of 239 .

For the dijet cross-sections we consider three Run I measurements from ATLAS and CMS, specifically the ATLAS and CMS $7 \mathrm{TeV}[16,18]$ double-differential distributions and the CMS $8 \mathrm{TeV}$ triple-differential distributions [19]. Note that currently ATLAS dijet measurements are only available at 7 and $13 \mathrm{TeV}$, but not at $8 \mathrm{TeV}$. The ATLAS data are double-differential in $m_{j j}$ and $\left|y^{*}\right|$. The corresponding ranges are $260 \mathrm{GeV} \leq m_{j j} \leq 4.27 \mathrm{TeV}$ and $0 \leq y^{*} \leq 3.0$. The CMS $7 \mathrm{TeV}$ data [16] are instead double-differential in $m_{j j}$ and $\left|y_{\max }\right|$. The ranges are $200 \mathrm{GeV} \leq m_{j j} \leq 5 \mathrm{TeV}$ and $0 \leq|y|_{\max } \leq 2.5$.

The CMS $8 \mathrm{TeV}$ [19] data are triple differential in $p_{T \text {,avg, }}$ $y_{b}$, and $\left|y^{*}\right|$. The ranges are $133 \mathrm{GeV} \leq p_{T, \text { avg }} \leq 1.78 \mathrm{TeV}$ and $0 \leq y_{b}, y^{*} \leq 3$.

For all these measurements, we will use the complete set of systematic uncertainties and correlations available from HEPDATA. Various correlation models, whereby specific systematic uncertainties are decorrelated to a different extent, have been proposed, depending on the dataset. As a representative example, we will study some of these models in the case of the ATLAS $7 \mathrm{TeV}$ and $8 \mathrm{TeV}$ single-inclusive jet cross-sections.

\section{Theoretical calculations and implementation}

In this section we present the main aspects of the theoretical computations on which our phenomenological studies are based. First we address QCD corrections, discuss the scale choice, and assess the size of NNLO corrections. Then we discuss EW corrections, assess their size, and explain how they are combined with QCD corrections for the purpose of PDF determination.

\subsection{QCD corrections}

Single-inclusive and dijet observables display a somewhat different perturbative behavior. We discuss the two observables in turn: for each of them, we present the dependence of results on the central scale, its optimal choice, and the NNLO corrections.

The single-inclusive jet cross-section is in general rather sensitive to the choice of central scale, even at NNLO. A detailed study of the scale dependence of the NNLO QCD 
predictions for single-inclusive jet production was carried out in [11], where three different scales (and their multiples) were discussed in detail: the individual jet transverse momentum $p_{T}$, the leading jet transverse momentum $p_{T, 1}$, and the scalar sum of the transverse momenta of all partons in the event

$$
\widehat{H}_{T}=\sum_{i \in \text { partons }} p_{T, i} \text {. }
$$

Note that $p_{T, 1}$ and $\widehat{H}_{T}$ are event-based choices, i.e. all jets in the event have the same scale, while $p_{T}$ is a jet-based choice, i.e. it is a property of the individual jet within a given event.

The commonly used scale choices $\mu=p_{T}$ or $\mu=p_{T, 1}$ lead to predictions which even at NNLO may differ by an amount which is comparable to, or larger than, their scale dependence [10], a behavior which was traced in Ref. [11] to the infrared sensitivity of the second-jet contribution, and which is aggravated by the choice $\mu=p_{T, 1}$. In Ref. [11] scale choices were thus compared according to a number of criteria: perturbative convergence; scale uncertainty as error estimate; perturbative convergence of the individual jet spectra; and stability of the second jet distribution. The eventbased scale $\mu=\widehat{H}_{T}$ and the jet-based scale $\mu=2 p_{T}$ were singled out as optimal choices. Here we will adopt $\mu=\widehat{H}_{T}$ as central scale choice; results obtained with this scale choice will be compared in Sect. 4 to those found using $\mu=p_{T}$, which was the baseline choice adopted in previous NNPDF determinations, specifically NNPDF3.1.

NNLO QCD corrections computed with NNLOJET [46] will be included by supplementing theoretical predictions accurate to NLO QCD with $K$-factors defined as

$K_{\mathrm{NNLO}}^{\mathrm{QCD}} \equiv \frac{\sum_{i j} \widetilde{\sigma}_{i j}^{\mathrm{NNLO}} \otimes \mathcal{L}_{i j}^{\mathrm{NNLO}}}{\sum_{i j} \widetilde{\sigma}_{i j}^{\mathrm{NLO}} \otimes \mathcal{L}_{i j}^{\mathrm{NNLO}}}$,

where the sum runs over partonic subchannels, $\widetilde{\sigma}_{i j}$ are partonic cross-sections, and $\mathcal{L}_{i j}$ the corresponding parton luminosities, computed both in the numerator and the denominator using NNPDF3.1 NNLO as a fixed input PDF set.

In Fig. 1 we show the NNLO QCD $K$-factors, Eq. (3.2), corresponding to the ATLAS $7 \mathrm{TeV}$ and CMS $8 \mathrm{TeV}$ singleinclusive jet cross-sections evaluated with the NNPDF3.1 NNLO PDF set and $\mu=\widehat{H}_{T}$ as central scale. Results are shown as a function of the jet $p_{T}$ in different jet rapidity bins, with the central (forward) bins in the left (right) plot. At central rapidities, the NNLO $K$-factor increases monotonically with $p_{T}$ from about $5 \%$ to about $20-25 \%$. This growth with $p_{T}$ becomes less marked as the jet rapidity increases: in fact at $8 \mathrm{TeV}$ for $|y| \geq 1.5$ the $K$-factor depends only mildly on the jet $p_{T}$. The $K$-factors display moderate point-to-point fluctuations, especially in the forward rapidity bins.

We now turn to dijets. A variety of scale choices is possible: two popular choices are the dijet invariant mass $m_{j j}$ and the average transverse momentum $p_{T, \text { avg }}$ of the two leading jets. Theoretical predictions computed with either of these scale choices differ significantly at NLO. This difference is substantially reduced at NNLO, with $\mu=m_{j j}$ emerging as a preferred choice, based on the criteria of perturbative convergence, and residual scale dependence of the NNLO prediction $[47,48]$. This is the scale choice which we will adopt in the sequel.

In Fig. 2 we display the NNLO QCD $K$-factors, Eq. (3.2), computed with this scale choice and the NNDPF3.1 NNLO PDF set, for the ATLAS $7 \mathrm{TeV}$ and CMS $8 \mathrm{TeV}$ dijet crosssections. For ATLAS, the $K$-factors at small rapidity separations are somewhat below unity for low invariant masses, then grow monotonically with $m_{j j}$ up to about $K \sim 1.15$ at the highest $m_{j j} \sim 4 \mathrm{TeV}$. For larger rapidity separations, $1.5 \leq\left|y^{*}\right| \leq 3.0$, the $K$-factors are less sensitive to $m_{j j}$, and their value corresponds to corrections between 10 and $20 \%$. For CMS, as previously mentioned, the measurement

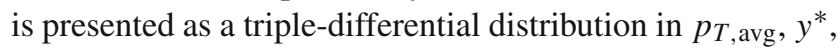
and $y_{b}$. As seen in Fig. 2, the qualitative behavior of the $K$ factors is similar in all rapidity bins, and shows a monotonic

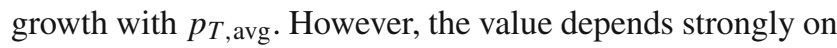
the rapidity difference, with the $K$-factor larger at larger $y^{*}$. For example, in the $0 \leq y_{b}, y^{*} \leq 1$ bin the $K$-factor ranges from a few percent at low $p_{T, \text { avg }}$ to up to $15 \%$, while in the $0 \leq y_{b} \leq 1$ and $2 \leq y^{*} \leq 3$ bin it goes up to $25 \%$. These $K$-factors display sizable point-to-point fluctuations.

\subsection{Electroweak corrections}

We have determined EW corrections for all of the singleinclusive jet and dijet datasets considered in this work by using the calculation of Ref. [49], suitably extended to the case of single-inclusive jets. The EW corrections computed in Ref. [49] include $O\left(\alpha \alpha_{s}\right)$ and $O\left(\alpha^{2}\right)$ tree level contributions (where $\alpha$ and $\alpha_{s}$ are the electromagnetic and strong couplings, respectively), and the weak radiative corrections of $O\left(\alpha \alpha_{s}^{2}\right)$. In particular, they include the virtual exchange of weak bosons that give rise to the dominant EW Sudakov logarithms, suitably combined with the respective hard QCD emissions to cancel infrared singularities. This is what will be referred to as EW corrections in the remainder of this paper.

We include EW corrections through a $K$-factor defined as

$K^{\mathrm{EW}} \equiv \frac{\sum_{i j} \widetilde{\sigma}_{i j}^{\mathrm{LO} \mathrm{QCD}+\mathrm{EW}} \otimes \mathcal{L}_{i j}^{\mathrm{NNLO}}}{\sum_{i j} \widetilde{\sigma}_{i j}^{\mathrm{LO}, \mathrm{QCD}} \otimes \mathcal{L}_{i j}^{\mathrm{NNLO}}}$,

where the partonic cross-sections in the numerator are obtained by combining the contributions computed in Ref. [49] with the LO QCD computation. The $K$-factor defined in Eq. (3.3) has been computed using a proprietary 

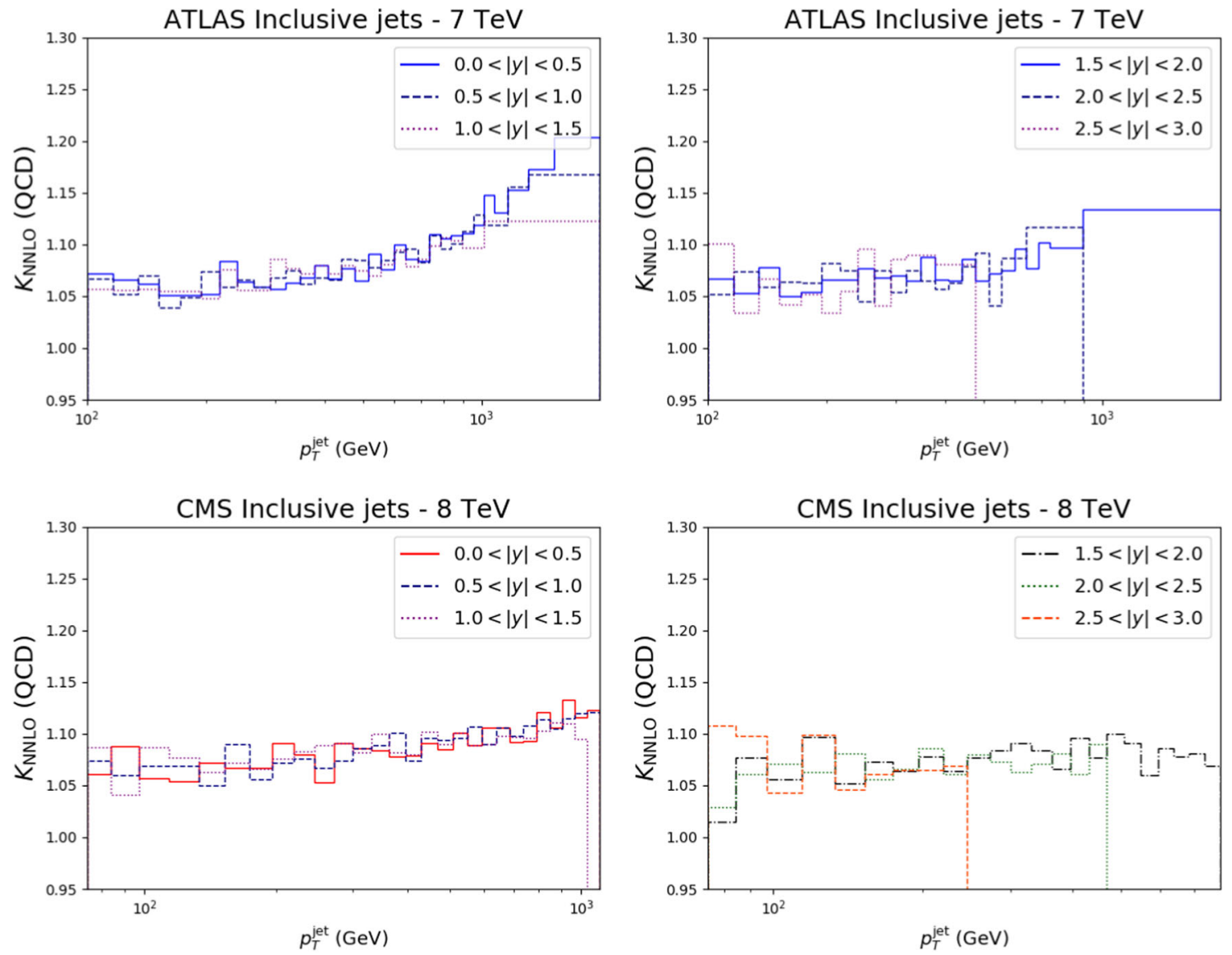

Fig. 1 The NNLO QCD $K$-factors, Eq. (3.2), for the ATLAS $7 \mathrm{TeV}$ (top) and CMS $8 \mathrm{TeV}$ (bottom) single-inclusive jet cross-sections evaluated using NNPDF3.1 PDFs and scale $\mu=\widehat{H}_{T}$. Results are shown as

a function of the jet $p_{T}$ in different jet rapidity bins, with the central (forward) bins shown in the left (right) plot

code [49]. Electroweak $K$-factors have been evaluated using consistently the NNPDF3.1 NNLO PDF set, and the same scale choice as that of the corresponding NNLO QCD predictions. Note that because of cancellations between (negative) Sudakov logarithms and (positive) subleading Born contributions, the $K$-factors are quite sensitive to the underlying parton decomposition, and it is consequently important to make a consistent choice of PDFs in the computation of QCD and EW $K$-factors.

The $K$-factors thus computed are shown in Fig. 3 for the ATLAS $7 \mathrm{TeV}$ and CMS $8 \mathrm{TeV}$ single-inclusive jet crosssections and for the ATLAS and CMS $7 \mathrm{TeV}$ dijet crosssections. Results are shown as a function of $p_{T}$ for singleinclusive jets and as a function of $m_{j j}$ for dijets, in bins of rapidity $y$ (single-inclusive), absolute rapidity difference $y^{*}$ (ATLAS dijets) or maximum absolute rapidity $y_{\max }$ (CMS dijets). In all cases the qualitative behavior is similar: the $K$ factor is close to unity for small values of $p_{T}$ or $m_{j j}$; it is flat (in fact slightly decreasing) for large values of the rapidity variable; and it grows with respectively $p_{T}$ or $m_{j j}$ at central

rapidity, the growth being stronger at smaller rapidity. The largest EW correction can reach $20 \%$ or more for transverse momenta or invariant masses in the $\mathrm{TeV}$ range and smaller rapidity.

\subsection{Implementation}

For each dataset, we produce fast interpolation grids, accurate to NLO in QCD, whereby partonic matrix elements are precomputed in such a way that the numerical convolution with generic input PDFs can be efficiently approximated by means of interpolation techniques. To this purpose, we use NLOJET++ [50] interfaced to FASTNLO [51]. The computation is performed with the scale choices discussed in Sect. 3.1, and it is benchmarked against the NNLOJET computation. These fast interpolation grids are then combined with PDF evolution kernels, in a format compliant with the NNPDF framework, using APFELGRID [52]. Such a combination is required to speed up the computation of hadronic observables when the fit is performed. 

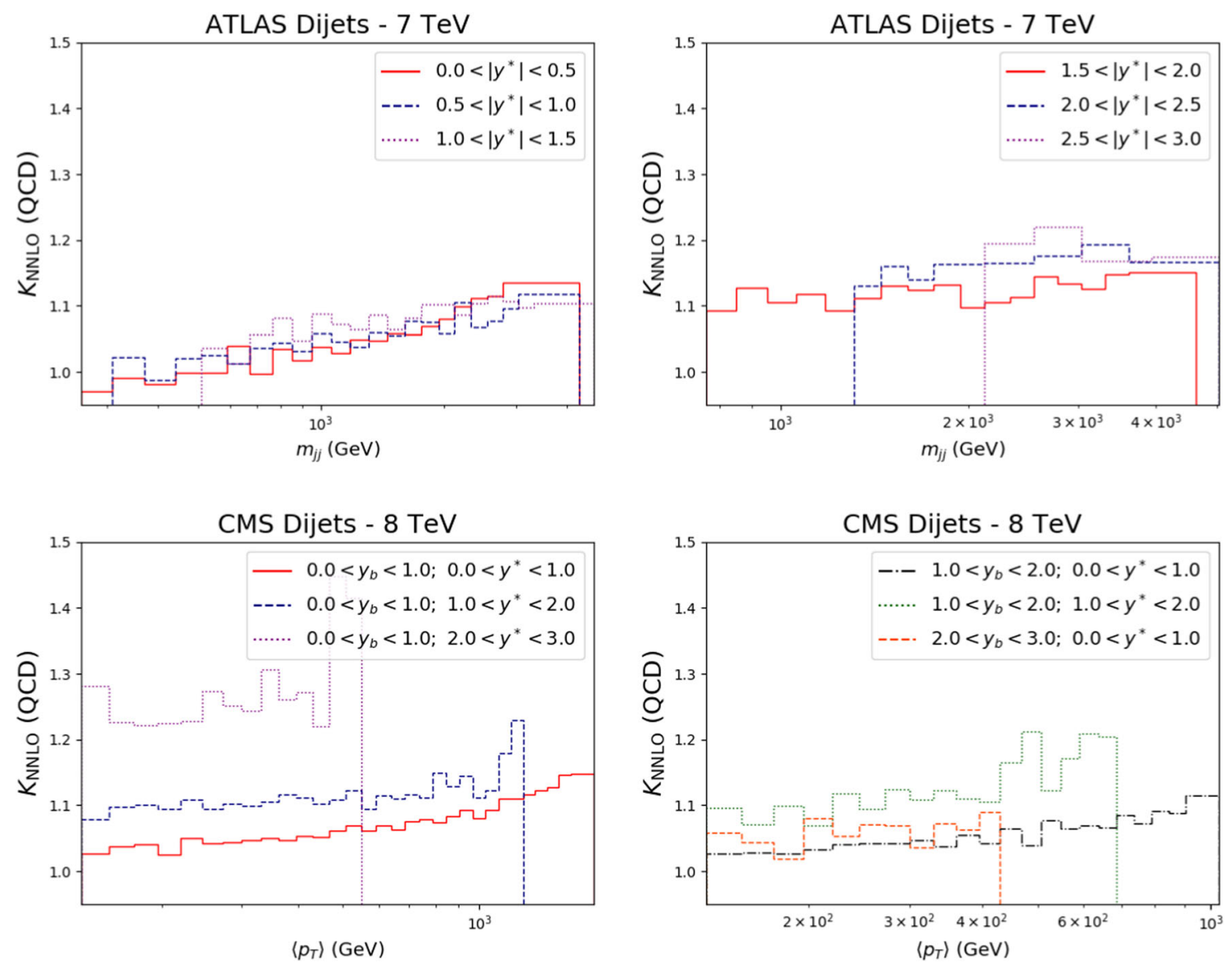

Fig. 2 The NNLO QCD $K$-factors, Eq. (3.2), corresponding to the ATLAS $7 \mathrm{TeV}$ (top) and CMS 8 TeV (bottom) dijet cross-sections evaluated with NNPDF3.1 PDF and scale $\mu=m_{j j}$. Results are shown as function of the jet $p_{T}$ in different jet rapidity bins, with the most central (forward) bins in the left (right) plot

Fast interpolation grids accurate to NNLO, for instance in the APPLFAST format, are not yet publicly available: indeed, the NNLOJET+APPLFAST fast interpolation tables with NNLO QCD corrections are so far only available for jet production in deep-inelastic scattering [53]. We therefore implement NNLO and EW corrections by supplementing our NLO grids with the QCD and EW $K$-factors defined above, which we combine through the multiplicative prescription

$$
\begin{aligned}
& \left.\frac{d^{2} \sigma}{d p_{T} d y}\right|_{\mathrm{NNLO}_{\mathrm{QCD}}+\mathrm{EW}}=\left.\frac{d^{2} \sigma}{d p_{T} d y}\right|_{\mathrm{NLO}_{\mathrm{QCD}}} \\
& \times K_{\mathrm{NNLO}}^{\mathrm{QCD}}\left(p_{T}, y, \sqrt{s}\right) \times K^{\mathrm{EW}}\left(p_{T}, y, \sqrt{s}\right) .
\end{aligned}
$$

The first term on the right-hand side of the equation is the output of the NLO computation, while the second and third terms are the bin-by-bin QCD and EW $K$-factors defined in Eqs. (3.2) and (3.3), respectively. If the EW $K$-factor is not included, Eq. (3.4) exactly reproduces the NNLO results obtained with NNLOJET.

As observed in Sects. 3.1-3.2, QCD $K$-factors are affected by point-to-point fluctuations which reveal an underlying numerical uncertainty. For illustration purposes, this uncertainty is displayed in Fig. 4 for the central rapidity bins of the ATLAS 7 TeV single-inclusive jet and of the CMS $8 \mathrm{TeV}$ dijet distributions. We have estimated this uncertainty through the procedure for the suppression of outliers as described in Ref. [54]. When performing PDF fits, this numerical uncertainty is added in quadrature to the experimental uncertainty, fully uncorrelated datapoint by datapoint. An alternative possibility would be to perform a smooth interpolation of the $K$-factor, see Ref. [55].

Finally, we note that the theoretical computations of single-inclusive and dijet observables are subject to nonperturbative corrections and to missing higher order uncertainties (MHOU). The former arise from the underlying event and multiple parton interactions, and are estimated by the experimental collaborations by comparing predictions obtained from different Monte Carlo parton shower generators. In the case of all of the CMS measurements, they are provided in terms of point-by-point rescaling factors, which we apply to the data together with an additional, fully correlated, systematic uncertainty, which we estimate as the dif- 

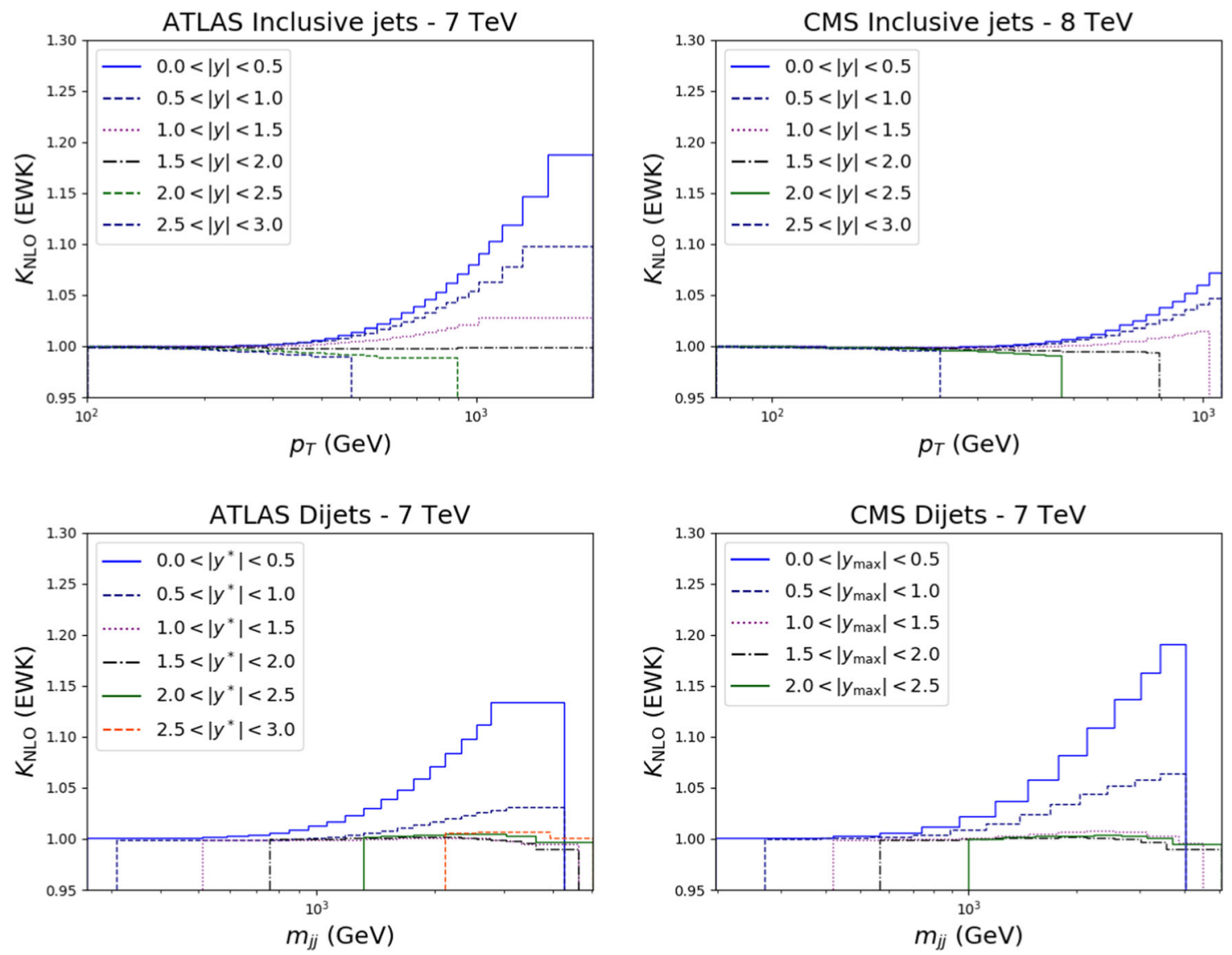

Fig. 3 The EW $K$-factors, Eq. (3.3), for the ATLAS and CMS singleinclusive (top) and dijet (bottom) measurements. For single-inclusive jets the $K$-factors are shown as a function of jet $p_{T}$ in six different

rapidity bins. For dijets they are shown as a function of the dijet invariant mass $m_{j j}$ for different $y^{*}$ bins for ATLAS (left) or $y_{\max }$ bins for CMS (right)
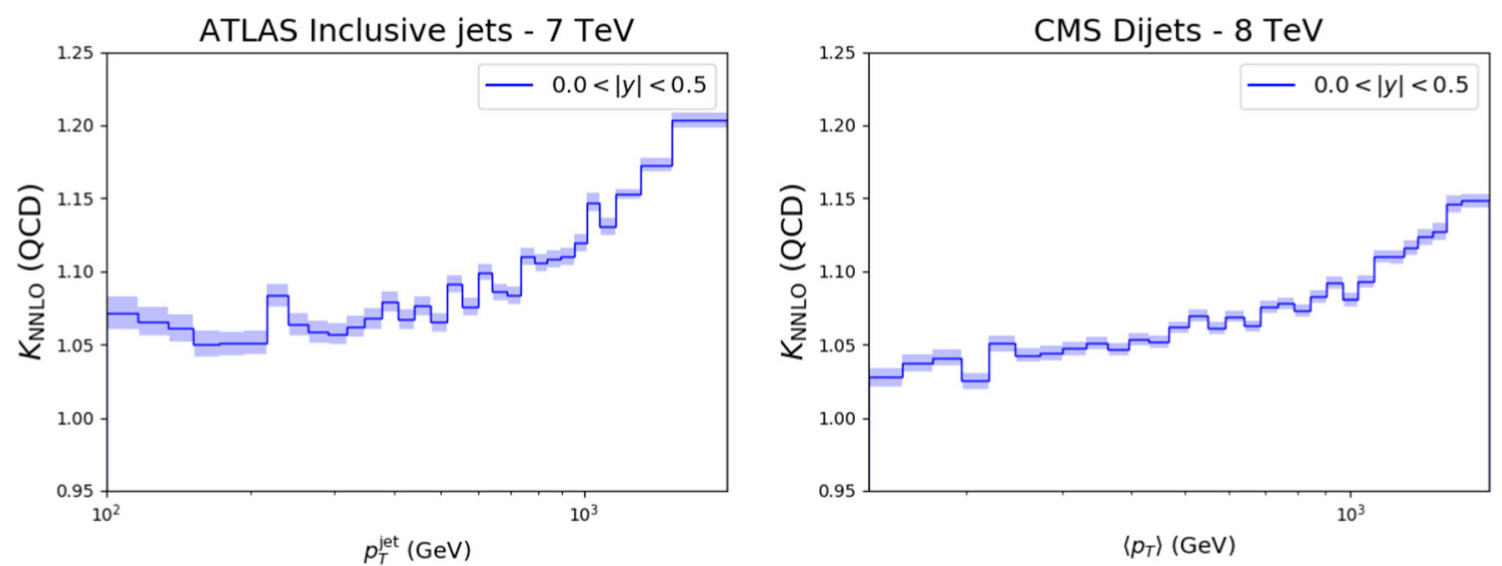

Fig. 4 The NNLO QCD $K$-factors for the central rapidity bins of the ATLAS 7 TeV single-inclusive jets (left) and CMS 8 TeV dijets (right), with the Monte Carlo numerical uncertainties shown as filled bands around the central result 
ference between the value at each datapoint before and after rescaling. The estimate of MHOUs requires some care, especially for single-inclusive jets. This is due to the fact that there are accidental cancellations which occur for values of the jet radius $R \sim 0.5$ which are close to the values adopted by ATLAS and CMS, where the NLO scale dependence evaluated in a standard way is artificially small $[12,13]$, and thus is not a good estimator of the MHOU. A more reliable estimate of the MHOU requires performing uncorrelated scale variation $[13,56]$. The inclusion of MHOU in PDF fits, though in principle possible using the formalism of Refs. [43,44], goes beyond the scope of this paper, and we will not consider it further.

\section{Results}

We now present our main results. They consist of a set of global PDF determinations, in which the NNPDF3.1 global dataset is supplemented by the single-inclusive jet and inclusive dijet data presented in Sect. 2.3: by comparing fit results, we study the impact of varying the jet observable, the data, and the theory settings. Specifically, we have performed fits including either single-inclusive or dijet data, in each case using either the full data set, or $7 \mathrm{TeV}$ data or $8 \mathrm{TeV}$ data only, and with theory at pure NLO QCD, pure NNLO QCD, or NNLO QCD supplemented by EW corrections as discussed in Sect. 3.2. For the single-inclusive $7 \mathrm{TeV}$ data we have also performed fits with alternative choices of central scale. Finally, for the ATLAS $7 \mathrm{TeV}$ and $8 \mathrm{TeV}$ single-inclusive jet data we have studied the effect of the treatment of correlated systematics. We will first present in Sect. 4.2 all PDF sets based on single-inclusive data, including variations of scale choice and decorrelation model, then in Sect. 4.3 PDF sets based on inclusive dijet data, and finally in Sect. 4.4 draw general comparative conclusions on the behavior of different observables at different perturbative orders.

\subsection{PDF sets}

The inclusion of jet data in a global NNPDF3.1-like PDF determination essentially impacts only the gluon PDF, as was shown in Ref. [20], while leaving other PDFs essentially unchanged. The impact of the jet data on the gluon PDF can be assessed by computing the correlation coefficient (as defined in Ref. [57], see also Ref. [58]) between each datapoint and the gluon. The correlations for the largest and smallest rapidity bins of the datasets of Table 1 are shown in Fig. 5, computed using the default baseline NNLO PDF set (before inclusion of the jet data, \#bn, see Table 2 below). Correlations are seen to be large or very large (up to almost one) for all $x \gtrsim 10^{-2}$. Interestingly, the choice of the rapidity variable $y_{\max }$ appears to maximize the range in which a large correlation with the gluon is observed.

The full list of PDF determinations that we will discuss is given in Table 2, together with an ID that will be used to identify them. In this and all subsequent tables and plots "jets" is short for single-inclusive jets. Each row corresponds to a different choice of dataset or methodological settings, while columns correspond to the theory adopted: QCD at NLO or NNLO, without or with EW corrections included. By NLO or NNLO we mean that jets have been consistently added with NLO or NNLO theory to the respective NLO or NNLO global fit; note that EW corrections instead are only included for the jet observable, with all other observables in the global fit computed using pure QCD theory.

The jet data of Table 1 are added to a baseline dataset, which essentially coincides with the NNPDF3.1 dataset. This dataset includes: fixed-target neutral-current (NC) DIS structure function data from NMC [59,60], SLAC [61] and BCDMS [62]; charged-current (CC) DIS structure function data from CHORUS [63] and NuTeV [64,65]; HERA data from their combined measurements [66], including charmproduction cross sections [67] and $b$-tagged structure functions [68,69]; fixed-target Drell-Yan data from E866 [70-72] and E605 [73]; collider Drell-Yan data from CDF [74] and D0 [75-77]; and Drell-Yan, inclusive gauge boson, and toppair production data from ATLAS [78-85], CMS [86-93] and $\mathrm{LHCb}$ [94-97]. In total this baseline dataset contains $n_{\text {dat }}=3813$ datapoints, see Ref. [20] for more details. The number of datapoints corresponding to the jet data included in the various fits of Table 2 is given in Tables 3 and 4 below.

In all of these fits, experimental systematic uncertainties are fully correlated across bins of different kinematic variables, while statistical uncertainties coming from the unfolding are correlated only across bins of transverse momentum (for jets) or invariant mass (for dijets), but not across rapidity bins. The possibility of removing some or all of these correlations will be discussed in Sect. 4.2.4 below. Multiplicative uncertainties are treated with the $t_{0}$-method [98], and all fits in Table 2 are iterated once to ensure convergence of the $t_{0}$ method and preprocessing (see Ref. [20] for more details).

For jet or dijet data, non-perturbative corrections are included by default, as are Monte Carlo uncertainties due to finite numerical precision of NNLO QCD $K$-factor computations (see Sect. 3 for details). The factorization and renormalization scales are by default taken to be $\mu=\widehat{H}_{T}$ for singleinclusive jets, and $\mu=m_{j j}$ for dijets (see the discussion in Sect. 3). An alternative choice of scale for single-inclusive jets will be considered in Sect. 4.2.3 below.

All the fits listed in Table 2 otherwise closely follow the NNPDF3.1 analysis [20]. Specifically, the same settings and codes are used for the computation of physical observables in the baseline dataset, and the same choice of kinematic cuts, of values of physical parameters, and of fitting methodology 

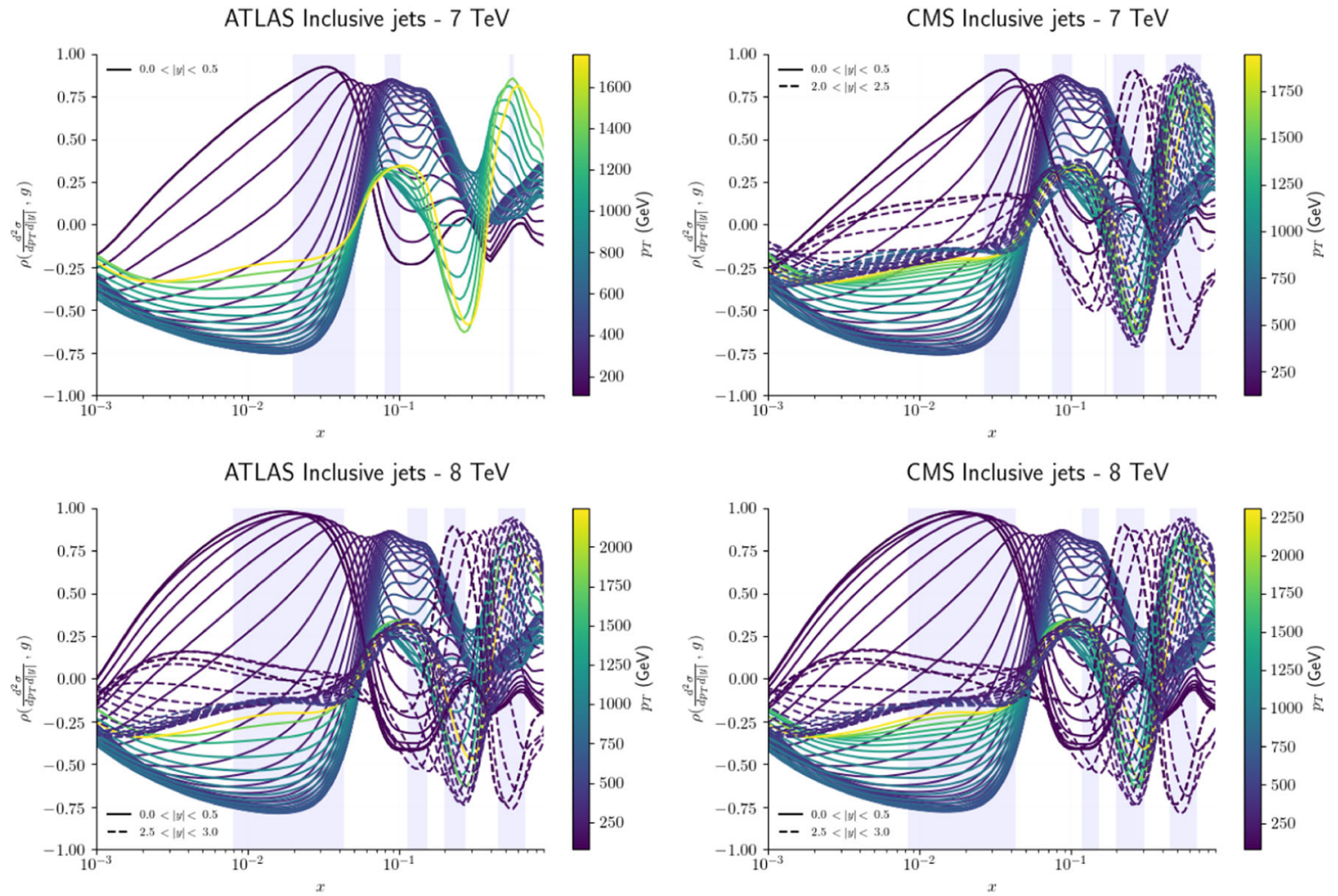

ATLAS Dijets - $7 \mathrm{TeV}$
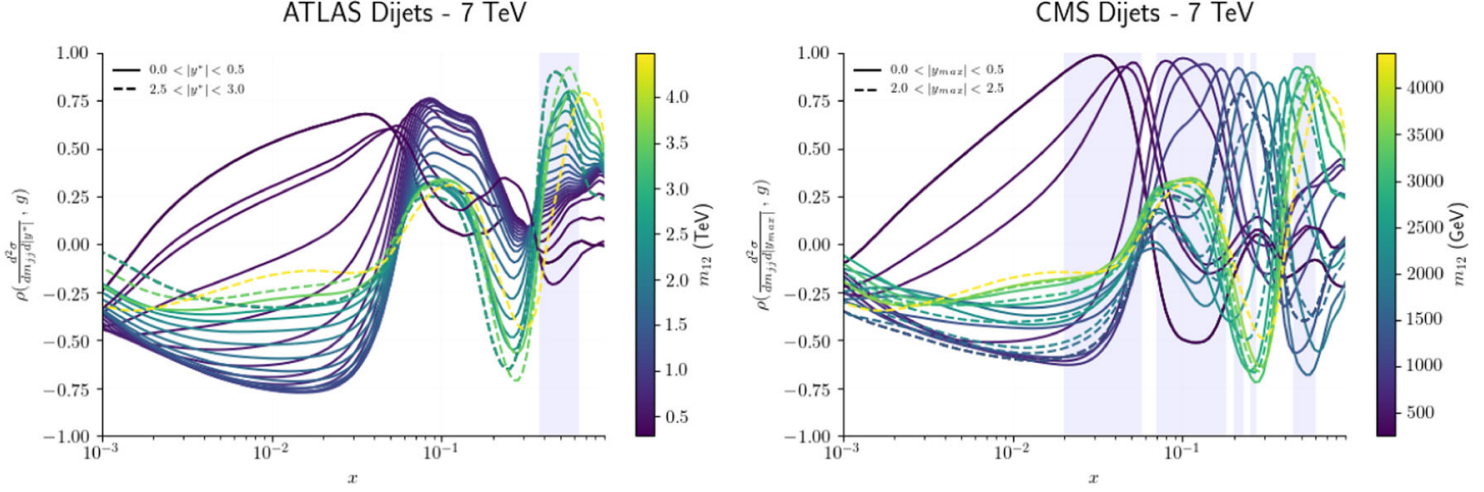

CMS Dijets - $8 \mathrm{TeV}$

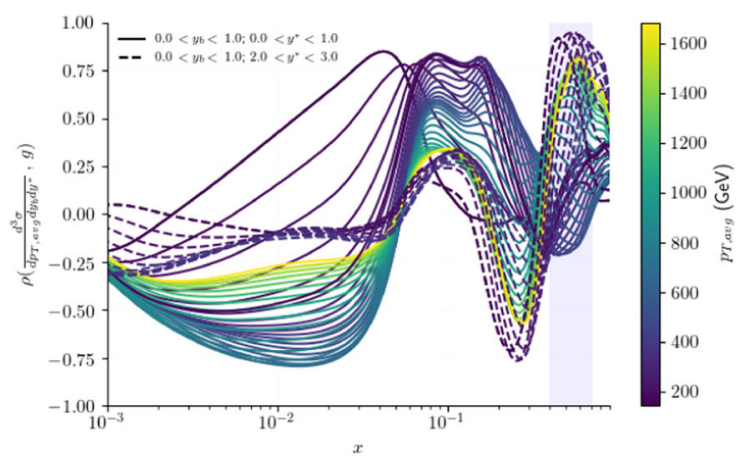

Fig. 5 Correlation coefficients between the data of Table 1 and the gluon PDF $g(x)$. Each curve corresponds to a different datapoint, with the value of $p_{T}$ corresponding to the color code on the right of the plot, and only curves for the points in the largest and smallest rapidity bins are shown. The shaded bands denote regions in which the maximum correlation is greater than $90 \%$ of the maximum correlation in the whole plot 
Table 2 The PDF determinations discussed in this study and their IDs. Each row corresponds to a different choice of input jet dataset or fit settings (listed in the first column), and each column corresponds to a different theory accuracy (listed in the first row). The ID encodes the process used ( $\mathrm{j}$ for single-inclusive jets and $\mathrm{d}$ for dijets); the data used (a for all, 7 or 8 for the $7 \mathrm{TeV}$ or $8 \mathrm{TeV}$ datasets); the perturbative accuracy (n for QCD NNLO, w if EW corrections included); and the choice of scale (pt when $\mu=p_{T}^{\text {jet }}$ ); special treatment of the ATLAS $7 \mathrm{TeV}$ data (cor for all bins included, correlated; dec, decorrelated; pcor, partially correlated); and special treatment of the ATLAS $8 \mathrm{TeV}$ data (dec for all bins included, decorrelated; pcor, partially correlated). In this and subsequent tables and plots "jets" is short for single-inclusive jets

\begin{tabular}{|c|c|c|c|}
\hline & $\mathrm{NNLO}_{\mathrm{QCD}}+\mathrm{EW}$ & $\mathrm{NNLO}_{\mathrm{QCD}}$ & $\mathrm{NLO}_{\mathrm{QCD}}$ \\
\hline Baseline (see text) & - & bn & $\mathrm{b}$ \\
\hline ATLAS and CMS jets $7-8 \mathrm{TeV}$ & janw & - & - \\
\hline ATLAS and CMS jets $7 \mathrm{TeV}$ & $\mathrm{j} 7 \mathrm{nw}$ & $\mathrm{j} 7 \mathrm{n}$ & $\mathrm{j} 7$ \\
\hline ATLAS and CMS jets $7 \mathrm{TeV}\left(\mu=p_{T}^{\text {jet }}\right)$ & - & j7n-pt & j7-pt \\
\hline ATLAS and CMS jets $8 \mathrm{TeV}$ & j8nw & j8n & j8 \\
\hline ATLAS and CMS jets 7-8 TeV (all ATLAS bins, full corr.) & janw-7cor & - & - \\
\hline ATLAS and CMS jets 7-8 TeV (all ATLAS bins $7 \mathrm{TeV}$, full decorr.) & janw-7dec & - & - \\
\hline ATLAS and CMS jets $7-8 \mathrm{TeV}$ (all ATLAS bins $7 \mathrm{TeV}$, part. corr.) & janw-7pcor & - & - \\
\hline ATLAS and CMS jets 7-8 TeV (all ATLAS bins $8 \mathrm{TeV}$, full decorr.) & janw-8dec & - & - \\
\hline ATLAS and CMS jets $7-8 \mathrm{TeV}$ (all ATLAS bins $8 \mathrm{TeV}$, part. corr.) & janw-8pcor & - & - \\
\hline ATLAS and CMS dijets 7-8 TeV & danw & - & - \\
\hline ATLAS and CMS dijets $7 \mathrm{TeV}$ & d7nw & $\mathrm{d} 7 \mathrm{n}$ & d7 \\
\hline CMS dijets $8 \mathrm{TeV}$ & d8nw & $\mathrm{d} 8 \mathrm{n}$ & $\mathrm{d} 8$ \\
\hline
\end{tabular}

are adopted. All PDF sets include $N_{\text {rep }}=100$ Monte Carlo replicas. The REPORTENGINE software [99] is used in the sequel to analyze each fit and compute various fit metrics. Specifically, we consider the $\chi^{2}$ of the theory prediction for each dataset or combinations of datasets, defined according to Eqs. (7)-(8) of Ref. [100], and the distance $d$ between pairs of fits (see e.g. Eq. (48) of Ref. [101] for its definition).

The values of the $\chi^{2}$ per datapoint for all fits with default settings at NLO and NNLO with or without EW corrections and single-inclusive jet or dijet data are collected in Tables 3 and 4 , respectively; $\chi^{2}$ values are shown for all data in the global dataset, grouped by process type (DIS NC, DIS CC, Drell-Yan, $Z p_{T}$, top pair) and for all jet data, both those which are and those which are not included in each fit. The values of $\chi^{2}$ per datapoint for all jet data (included or not included) for all fits performed with alternative choices of central scale or alternative decorrelation models are collected in Table 5. In these tables, $\chi^{2}$ values corresponding to data not included in each fit are enclosed in square brackets.

\subsection{Single-inclusive jets}

We first present PDF sets obtained by including singleinclusive jet data. We discuss in turn the impact and consistency of individual datasets; perturbative QCD stability and the impact of EW corrections; the choice of central scale; and alternative data treatment and decorrelation models for the ATLAS $7 \mathrm{TeV}$ data.

\subsubsection{Impact and consistency of datasets}

We provide a general comparative assessment of the impact of single-inclusive jet data on PDFs by comparing fits performed with the default theory settings of Sect. 3 and the highest theory accuracy, i.e. NNLO QCD theory for jet data and the rest of the global fit, and EW corrections included in the jet predictions only. According to the data included, these correspond to the fits \#bn, \#janw, \#j7nw, and \#j8nw of Table 2.

First, we compare fit \#janw, that contains all of the singleinclusive jet data, to the baseline \#bn, which does not include any jet data. Note that, as discussed in Sect. 2.3, in our default global dataset only the central rapidity bin of the ATLAS $7 \mathrm{TeV}$ data is included. Fits in which the full $7 \mathrm{TeV}$ ATLAS dataset is included will be discussed in Sect. 4.2.4 below. In Fig. 6 we display the distance between the PDF central values for the two fits, and the gluon PDF in both fits, normalized to the baseline, both at $Q=100 \mathrm{GeV}$. Recall that the distance $d$ is the difference in units of the standard deviation $\sigma$ of the mean, so for a sample of 100 replicas $d \sim 1$ corresponds to statistically identical PDFs (replicas extracted from the same underlying distribution) and $d \sim 10$ corresponds to PDFs that differ by one sigma. From Table 3 , we note that individual jet datasets are well described (with $\chi^{2}$ per datapoint of order one), except the $8 \mathrm{TeV}$ ATLAS data $\left(\chi^{2}=3.22\right)$, to be investigated in greater detail below. In comparison to the baseline fit, the inclusion of the single-inclusive jet data leads to a slight deterioration in the description of the ATLAS top pair rapidity distributions, whose $\chi^{2}$ per datapoint increases from 
Table 3 The $\chi^{2}$ per datapoint for all fits of Table 2 including singleinclusive jet data, with default settings. Results are shown for all datasets, aggregated by process type. For jets data, results are shown both for the sets included in each fit, and also for those not included, enclosed in square brackets. Combined results are also shown for all single-inclusive jet and for all dijet data, both for the full set, and for those included in each fit. The number of datapoints in each dataset is also shown

\begin{tabular}{|c|c|c|c|c|c|c|c|c|c|c|}
\hline Dataset & $n_{\text {dat }}$ & $\mathrm{b}$ & bn & janw & $\mathrm{j} 7$ & $\mathrm{j} 7 \mathrm{n}$ & j7nw & j8 & $\mathrm{j} 8 \mathrm{n}$ & j8nw \\
\hline DIS NC & 2103 & 1.17 & 1.17 & 1.18 & 1.17 & 1.18 & 1.17 & 1.17 & 1.17 & 1.18 \\
\hline DIS CC & 989 & 1.06 & 1.10 & 1.11 & 1.06 & 1.11 & 1.10 & 1.08 & 1.11 & 1.11 \\
\hline Drell-Yan & 577 & 1.35 & 1.33 & 1.30 & 1.35 & 1.31 & 1.31 & 1.34 & 1.31 & 1.31 \\
\hline$Z p_{T}$ & 120 & 1.84 & 1.01 & 1.02 & 1.85 & 1.02 & 1.02 & 1.89 & 1.03 & 1.03 \\
\hline Top pair & 24 & 1.10 & 1.05 & 1.25 & 1.09 & 1.06 & 1.02 & 2.00 & 1.61 & 1.24 \\
\hline $\operatorname{ATLAS} \sigma_{t \bar{t}}$ & 3 & 2.02 & 0.90 & 0.70 & 1.68 & 0.74 & 0.72 & 1.70 & 0.79 & 0.78 \\
\hline ATLAS $t \bar{t}$ rap & 9 & 1.12 & 1.22 & 2.01 & 1.25 & 1.38 & 1.31 & 2.93 & 2.78 & 1.96 \\
\hline $\mathrm{CMS} \sigma_{t \bar{t}}$ & 3 & 0.53 & 0.22 & 0.21 & 0.42 & 0.24 & 0.31 & 0.34 & 0.17 & 0.19 \\
\hline CMS $t \bar{t}$ rap & 9 & 0.98 & 1.17 & 0.98 & 0.96 & 1.09 & 1.04 & 1.65 & 1.12 & 0.99 \\
\hline Jets (all) & 520 & [1.48] & {$[2.60]$} & 1.88 & [1.86] & {$[2.45]$} & {$[2.53]$} & {$[1.20]$} & {$[1.75]$} & [1.89] \\
\hline Jets (fitted) & & - & - & 1.88 & 0.79 & 1.15 & 1.12 & 1.40 & 2.05 & 2.20 \\
\hline ATLAS $7 \mathrm{TeV}$ & 31 & {$[1.26]$} & {$[1.87]$} & 1.59 & 1.12 & 1.73 & 1.15 & [1.07] & [1.69] & [1.62] \\
\hline ATLAS $8 \mathrm{TeV}$ & 171 & {$[2.60]$} & {$[5.01]$} & 3.22 & {$[3.55]$} & {$[4.76]$} & [4.58] & 2.03 & 3.18 & 3.25 \\
\hline CMS 7 TeV & 133 & {$[0.60]$} & {$[1.06]$} & 1.09 & 0.71 & 1.01 & 1.11 & {$[0.72]$} & [0.94] & [1.14] \\
\hline CMS $8 \mathrm{TeV}$ & 185 & {$[1.10]$} & [1.59] & 1.25 & {$[1.24]$} & {$[1.47]$} & {$[1.80]$} & 0.81 & 1.01 & 1.23 \\
\hline Dijets (all) & 266 & [3.49] & {$[3.07]$} & {$[2.10]$} & [4.16] & {$[2.96]$} & {$[2.56]$} & {$[3.34]$} & {$[2.21]$} & [2.22] \\
\hline Dijets (fitted) & & - & - & - & - & - & - & - & - & - \\
\hline ATLAS $7 \mathrm{TeV}$ & 90 & [1.49] & {$[2.47]$} & [1.95] & {$[1.77]$} & {$[2.46]$} & [1.97] & {$[1.43]$} & {$[2.28]$} & [2.01] \\
\hline CMS $7 \mathrm{TeV}$ & 54 & {$[2.06]$} & {$[2.40]$} & {$[2.08]$} & {$[2.43]$} & {$[2.50]$} & {$[2.12]$} & {$[1.65]$} & {$[2.00]$} & [2.15] \\
\hline CMS $8 \mathrm{TeV}$ & 122 & {$[5.60]$} & {$[3.81]$} & {$[2.21]$} & {$[6.70]$} & {$[3.53]$} & {$[3.20]$} & {$[5.48]$} & [2.26] & [2.39] \\
\hline Total & & 1.20 & 1.18 & 1.28 & 1.17 & 1.17 & 1.17 & 1.39 & 1.27 & 1.27 \\
\hline
\end{tabular}

1.22 to 2.01. On the other hand, it leads to an improvement in the description of the dijet data, especially the $8 \mathrm{TeV}$ CMS data, which are not included in any of these fits. This suggests that the inclusion of single-inclusive and dijet data have a similar impact on PDFs, as we shall also see in Sect. 4.3.1 and discuss in greater detail in Sect. 4.4 below.

As mentioned above, and as it is clear from the distance plot in Fig. 6, single-inclusive jet data only have an impact on the gluon. The regions which are most affected are $x \simeq 0.05$, $0.1 \lesssim x \lesssim 0.2$, and $0.3 \lesssim x \lesssim 0.5$, consistently with the correlation plots of Fig. 5: in these regions the gluon PDF changes by up to slightly more than half sigma. In comparison to the baseline, the central gluon PDF is suppressed by about $2 \%$ in the small $x$ region and enhanced by about $4 \%$ in the large $x$ regions, though it always remains within the uncertainty band of the baseline.

We next assess the relative impact of different jet datasets, by adding to the comparison of the baseline (\#bn) and the fit with all single-inclusive jet data (\#janw) also fits in which only $7 \mathrm{TeV}$ (\#j7nw) or $8 \mathrm{TeV}$ (\#j8nw) jet data are included, all with the same settings (NNLO QCD+EW). The comparison is shown in Fig. 7, where we compare the gluon and its relative uncertainty. Here and henceforth, when comparing relative uncertainties, the uncertainties shown are computed as a ratio to a common baseline, i.e. the plot displays all uncertainties as a percentage of the same reference fit. From Table 3, we note that the unsatisfactory description of the ATLAS $8 \mathrm{TeV}$ data persists even when the $7 \mathrm{TeV}$ data are not included in the fit, and the deterioration in fit quality for the ATLAS top data in the global fit is also similar. On the other hand, the fit in which only $7 \mathrm{TeV}$ jets are included shows excellent fit quality both for the jet data and the global dataset. A significant difference between these two datasets is that for the $7 \mathrm{TeV}$ data only the central rapidity bin is included, while for the $8 \mathrm{TeV}$ data all rapidity bins are included: this suggests that the $8 \mathrm{TeV}$ data may also be affected by similar issues in the treatment of correlations between rapidity bins. We will see in Sect. 4.2.4 that this is indeed the case.

The relative pull of the jet datasets at $7 \mathrm{TeV}$ and $8 \mathrm{TeV}$ can be inferred from Fig. 7. They both lead to a comparable suppression of the gluon PDF of about $1 \%$ in the region $0.3 \lesssim x \lesssim 0.5$, while they respectively enhance it by $4 \%$ and $2 \%$ in the region $0.1 \lesssim x \lesssim 0.2$. However, the decrease in gluon uncertainty is rather more marked upon inclusion of the $8 \mathrm{TeV}$ data, and in fact, results obtained including all jet data, or only $8 \mathrm{TeV}$ are almost identical. Specifically, in 
Table 4 Same as Table 3, but now for dijets. The baseline is repeated for ease of reference

\begin{tabular}{|c|c|c|c|c|c|c|c|c|c|c|}
\hline Dataset & $n_{\text {dat }}$ & $\mathrm{b}$ & bn & danw & d7 & $d 7 n$ & $\mathrm{~d} 7 \mathrm{nw}$ & $\mathrm{d} 8$ & $\mathrm{~d} 8 \mathrm{n}$ & $\mathrm{d} 8 \mathrm{nw}$ \\
\hline DIS NC & 2103 & 1.17 & 1.17 & 1.18 & 1.17 & 1.17 & 1.17 & 1.21 & 1.18 & 1.18 \\
\hline DIS CC & 989 & 1.06 & 1.10 & 1.12 & 1.07 & 1.09 & 1.09 & 1.11 & 1.11 & 1.12 \\
\hline Drell-Yan & 577 & 1.35 & 1.33 & 1.29 & 1.36 & 1.33 & 1.32 & 1.32 & 1.28 & 1.28 \\
\hline$Z p_{T}$ & 120 & 1.84 & 1.01 & 1.07 & 1.85 & 1.03 & 1.03 & 2.06 & 1.07 & 1.08 \\
\hline Top pair & 24 & 1.10 & 1.05 & 1.14 & 1.16 & 1.06 & 1.04 & 1.57 & 1.34 & 1.26 \\
\hline $\operatorname{ATLAS} \sigma_{t \bar{t}}$ & 3 & 2.02 & 0.90 & 0.66 & 1.79 & 0.74 & 0.73 & 0.80 & 0.68 & 0.69 \\
\hline ATLAS $t \bar{t}$ rap & 9 & 1.12 & 1.22 & 1.57 & 1.26 & 1.34 & 1.32 & 2.41 & 2.02 & 1.82 \\
\hline $\mathrm{CMS} \sigma_{t \bar{t}}$ & 3 & 0.53 & 0.22 & 0.53 & 0.48 & 0.29 & 0.28 & 0.01 & 0.74 & 0.67 \\
\hline CMS $t \bar{t}$ rap & 9 & 0.98 & 1.17 & 1.04 & 1.07 & 1.09 & 1.07 & 1.42 & 1.04 & 1.04 \\
\hline Jets (all) & 520 & {$[1.48]$} & {$[2.60]$} & {$[2.06]$} & [1.62] & {$[2.75]$} & {$[2.70]$} & [1.42] & [1.94] & [2.14] \\
\hline Jets (fitted) & & - & - & - & - & - & - & - & - & - \\
\hline ATLAS $7 \mathrm{TeV}$ & 31 & [1.26] & [1.87] & [1.63] & [1.26] & [1.86] & {$[1.74]$} & {$[1.00]$} & {$[1.70]$} & [1.61] \\
\hline ATLAS $8 \mathrm{TeV}$ & 171 & {$[2.60]$} & {$[5.01]$} & [3.36] & [2.62] & [4.80] & {$[4.65]$} & [2.18] & [3.30] & [3.55] \\
\hline CMS $7 \mathrm{TeV}$ & 133 & {$[0.60]$} & {$[1.06]$} & [1.06] & {$[0.71]$} & [1.13] & [1.14] & {$[0.77]$} & {$[0.97]$} & [1.07] \\
\hline CMS $8 \mathrm{TeV}$ & 185 & [1.10] & [1.59] & [1.64] & [1.42] & [2.16] & {$[2.17]$} & [1.27] & {$[1.41]$} & [1.68] \\
\hline Dijets (all) & 266 & [3.49] & {$[3.07]$} & 1.65 & [3.03] & {$[2.21]$} & [2.16] & [2.38] & {$[1.74]$} & [1.71] \\
\hline Dijets (fitted) & & - & - & 1.65 & 1.33 & 1.79 & 1.72 & 3.69 & 1.59 & 1.68 \\
\hline ATLAS $7 \mathrm{TeV}$ & 90 & [1.49] & {$[2.47]$} & 1.76 & 1.20 & 1.94 & 1.78 & [1.04] & [1.96] & [1.78] \\
\hline CMS $7 \mathrm{TeV}$ & 54 & {$[2.06]$} & {$[2.40]$} & 1.60 & 1.54 & 1.55 & 1.63 & [1.67] & {$[1.70]$} & [1.66] \\
\hline CMS $8 \mathrm{TeV}$ & 122 & {$[5.60]$} & {$[3.81]$} & 1.58 & {$[5.03]$} & {$[2.70]$} & {$[2.67]$} & 3.69 & 1.59 & 1.68 \\
\hline Total & & 1.20 & 1.18 & 1.22 & 1.33 & 1.20 & 1.19 & 1.33 & 1.20 & 1.20 \\
\hline
\end{tabular}

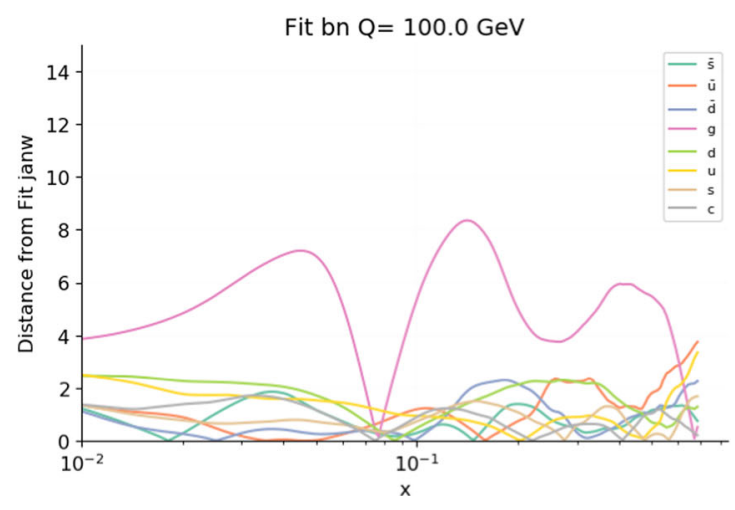

Fig. 6 Comparison between the baseline fit with no jet data (\#bn) and the fit with all single-inclusive jet data included (\#janw), both with default settings and the most accurate theory (NNLO QCD, including EW corrections for jets). The distance (see text) between all PDFs (left)

comparison to the baseline, inclusion of the $8 \mathrm{TeV}$ data results in a reduction of the relative gluon uncertainty at $x \simeq 0.2$ from 4 to $1.5 \%$, to be compared to the reduction 4 to $3 \%$ when the $7 \mathrm{TeV}$ data are included. A similar behavior was observed in the recent CT18 global PDF determination [22], which includes the ATLAS and CMS jet datasets at $7 \mathrm{TeV}$ and the CMS jet dataset at $8 \mathrm{TeV}$.

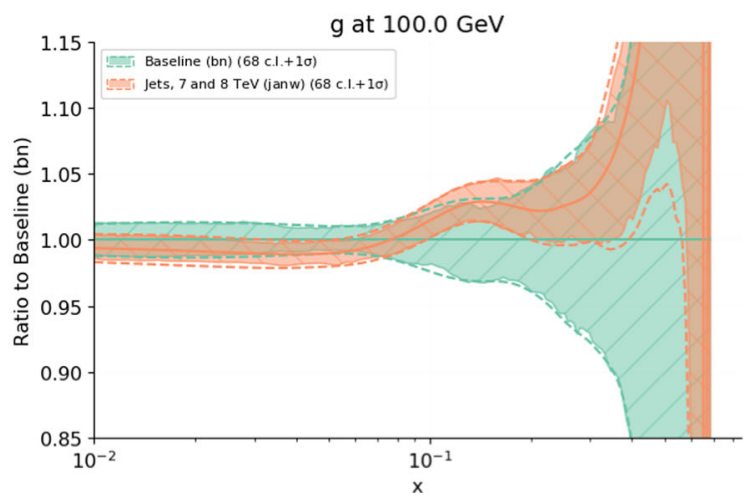

and the ratio of the gluon PDF to the baseline (right) are shown at the scale $Q=100 \mathrm{GeV}$. The shaded band is the $68 \%$ confidence interval, while the dashed lines are the edge of one sigma interval

\subsubsection{Impact of higher-order $Q C D$ and EW corrections}

Having assessed the impact of various single-inclusive jet data on PDFs with optimal theory settings, we now turn to the assessment of the perturbative stability of results. To this purpose, we compare fits at NLO, NNLO and with EW corrections (included for jet data only). The comparison is performed separately for the $7 \mathrm{TeV}$ and $8 \mathrm{TeV}$ data (fits \#j7, $\# j 7 \mathrm{n}, \# \mathrm{j} 7 \mathrm{nw}$; and $\# \mathrm{j} 8, \# \mathrm{j} 8 \mathrm{n}, \# \mathrm{j} 8 \mathrm{nw}$ respectively) both in oder 


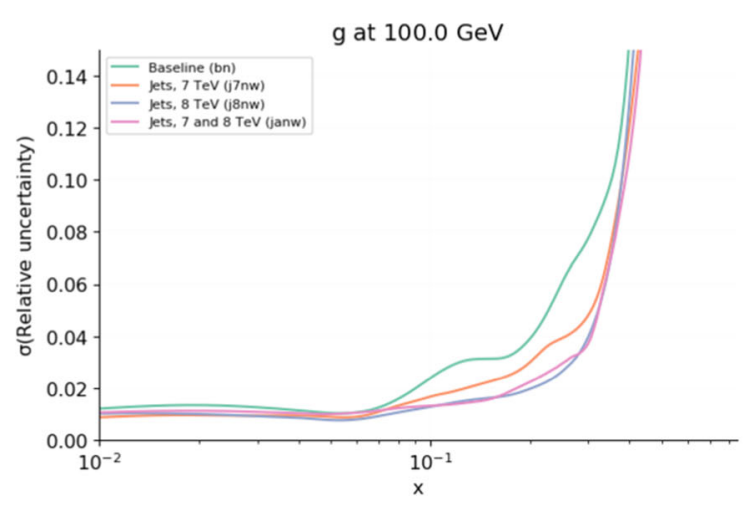

Fig. 7 Comparison between the baseline fit with no jet data (\#bn), and the fits with only $7 \mathrm{TeV}(\# \mathrm{j} 7 \mathrm{nw})$ or only $8 \mathrm{TeV}(\# \mathrm{j} 8 \mathrm{nw})$ jet data included. The relative uncertainty on the gluon PDF (left) and the ratio

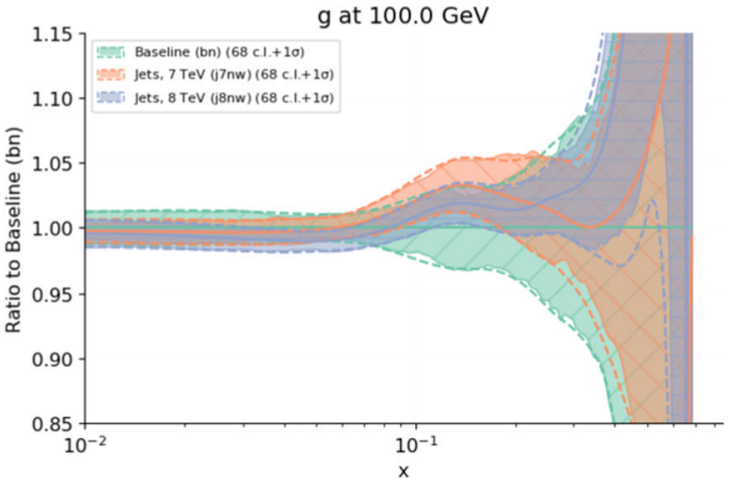

of the gluon PDF to the baseline (right) are shown at $Q=100 \mathrm{GeV}$. All results are shown as ratios to the baseline
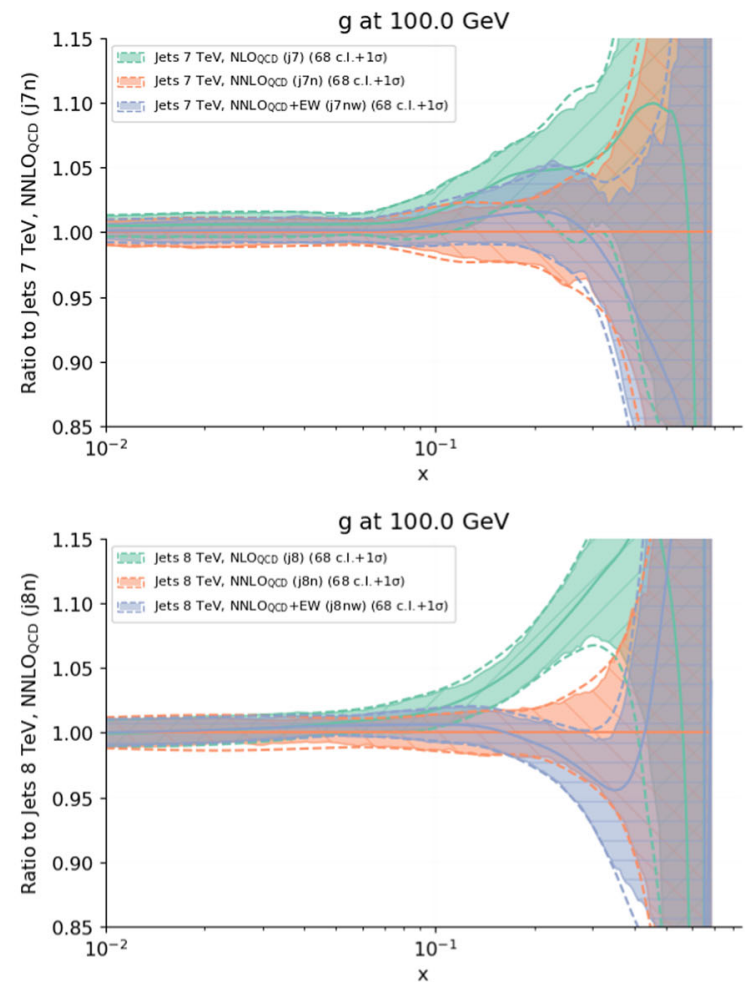

Fig. 8 Same as Fig. 7, but now comparing fits with NLO, NNLO and NNLO+EW theory for $7 \mathrm{TeV}$ (top: fits \#j7, \#j7n, \#j7nw respectively) and $8 \mathrm{TeV}$ (bottom: fits $\# \mathrm{j} 8, \# \mathrm{j} 8 \mathrm{n}$, \#j8nw). All results are now shown as ratios to the NNLO fit

to check consistency and to get a more detailed picture of the impact of different datasets. The gluon PDFs at $Q=$ $100 \mathrm{GeV}$ and their uncertainty for these fits are compared in Fig. 8.

It is clear from the figure that, both for $7 \mathrm{TeV}$ and $8 \mathrm{TeV}$ data, at NLO the gluon undergoes a significant distortion in the region $0.1 \lesssim x \lesssim 0.5$ in comparison to the NNLO results shown in Fig. 7. Specifically, at the peak, $x \sim 0.3$ the NLO gluon turns out to be by $30-40 \%$ larger than the baseline. This effect is driven by the jet data: we have verified that in the baseline (without jet data) the NLO gluon does show some distortion in comparison to the NNLO baseline, but by a much smaller amount, with the largest enhancement of order 5\%. This is thus evidence for large missing NNLO corrections to the single-inclusive jet cross section in the NLO fit. The effect is more pronounced for the $8 \mathrm{TeV}$ data, which can be understood as a consequence of their greater precision.

The effect of EW corrections is rather more moderate, with the shift of the central value always within the NNLO uncer- 
tainty band. Also, EW corrections seem to have an opposite effect when added to the fit to the $7 \mathrm{TeV}$ or the $8 \mathrm{TeV}$ data, leading to a slight enhancement of the gluon in the former case and a significant suppression for $x \gtrsim 0.2$ in the latter case. For both datasets, the uncertainty on the gluon for $x \gtrsim 0.1$, where the jet data have an impact, is reduced by a non-negligible amount by the inclusion of NNLO corrections. On the other hand the impact of the EW corrections is less clear. All this suggests that NNLO corrections have a significant impact, by affecting the best-fit large- $x$ gluon shape and improving its precision, while the impact of EW corrections is minor, and not clear-cut.

The effect of the inclusion of the NNLO and EW corrections on fit quality is less clear. Indeed, from Table 3, we observe that generally the fit quality to jet data deteriorates somewhat upon inclusion of NNLO corrections, and a little more upon inclusion of EW corrections. On the other hand, the global fit quality, as measured by the total $\chi^{2}$, is unchanged for the $7 \mathrm{TeV}$ data, and it improves significantly, from 1.39 to 1.27 , for the more precise $8 \mathrm{TeV}$ data, with the improvement mostly driven by the top and $Z p_{T}$ data which are most sensitive to the gluon. However, as already noted in Sect. 4.2.1, the $\chi^{2}$ of the top data deteriorates when adding the jet data to the baseline, and the fit quality to the ATLAS $8 \mathrm{TeV}$ data remains unchanged. This suggests that, for the more precise $8 \mathrm{TeV}$ data, the NNLO corrections reduce a tension between top and jets (especially ATLAS).

In summary, we conclude that, consistently with previous theoretical investigations $[11,12]$ NNLO corrections have a sizable impact on single-inclusive jets, and in particular their inclusion leads to a reduction of the uncertainty on the large$x$ gluon PDF and an improved consistency of the jet data with the rest of the global dataset, demonstrated by a reduction of the shift of the gluon central value upon inclusion of jets, and as an improvement of the global $\chi^{2}$ (for the more precise $8 \mathrm{TeV}$ jet data), when going from NLO to NNLO. Electroweak corrections do not appear to lead to improvements either in terms of fit quality or PDF uncertainty.

\subsubsection{Impact of the choice of scale}

We now turn to an assessment of the impact of the choice of central scale: specifically, we compare results obtained by fitting with our default scale choice $\mu=\widehat{H}_{T}$, chosen as optimal based on the studies of Ref. [11], and with the scale choice $\mu=p_{T}^{\text {jet }}$ used in NNPDF3.1 [20] and previous NNPDF studies. For ease of comparison to Ref. [20], the comparison is performed for fits to the $7 \mathrm{TeV}$ data (fits \# 7 and $\#$ j7-pt at NLO, and \#j7n and \#j7n-pt at NNLO). In Fig. 9 we show the distance between PDF central values of the two pairs of fits, at NLO and NNLO, and compare the corresponding gluon PDFs.
Inspection of Table 5 shows that at NLO the scale choice $\mu=\widehat{H}_{T}$ leads to a better description of the jet data, both included and not included in the fits, with respect to $\mu=p_{T}^{\mathrm{jet}}$. However, the effect of the scale choice on the PDFs is very mild (see Fig. 9), with a localized modification of the gluon below the half sigma level for $x \simeq 0.2$ and no effect on the other PDFs. On the other hand, at NNLO the two scale choices lead to almost indistinguishable results, both in terms of fit quality and PDF shape, with the scale choice $\mu=\widehat{H}_{T}$ leading to a slightly better description of data not included in the fit, and a difference in gluon central values barely above statistical indistinguishability.

We conclude that the scale choice $\mu=\widehat{H}_{T}$ is perturbatively more stable, in that it leads to a better NLO fit, but that at NNLO the choice of central scale is not an issue. Both conclusions are in agreement with the findings of Ref. [11].

\subsubsection{Impact of the choice of correlation models}

We finally discuss the impact of different correlation models on the ATLAS single-inclusive jet data. As repeatedly mentioned, only the central rapidity bin of the ATLAS $7 \mathrm{TeV}$ data was included in NNPDF3.1 and thus in our default fit because it was not possible to obtain a good fit when all rapidity bins were included, yet PDFs fitted to each rapidity bin turned out to be very close to each other $[20,45]$ : this suggests issues in the covariance matrix for these data, as extensively discussed in Ref. [24]. Further, as shown in Sect. 4.2.1, the corresponding ATLAS $8 \mathrm{TeV}$ data appear to be fully consistent with the $7 \mathrm{TeV}$ data, yet lead to a poor $\chi^{2}$ when included in the global fit, which suggests that they may suffer from a similar problem.

Here we will first check that indeed the inclusion of all rapidity bins from the $7 \mathrm{TeV}$ ATLAS data does not change the results for the PDFs, as argued in Refs. [20,45], but now by fitting all rapidity bins simultaneously, rather than one at a time as in Ref. [45], and with the new scale choice and jet dataset adopted here. We will then address the issue of the impact of the choice of correlation model, in particular by decorrelating different rapidity bins as suggested in Ref. [24] for the $7 \mathrm{TeV}$ data and in Ref. [15] for the $8 \mathrm{TeV}$ data.

To this purpose, we have performed five variant fits of our most accurate fit with default settings (\#janw) in which alternative treatments of the $7 \mathrm{TeV}$ or $8 \mathrm{TeV}$ ATLAS data are considered in turn (see Table 5). Concerning the $7 \mathrm{TeV}$ data, in a first fit (\#janw-7cor) all ATLAS rapidity bins are included: so in this fit the $7 \mathrm{TeV}$ and $8 \mathrm{TeV}$ are treated on an equal footing, with all bins included and correlated systematics treated using the published covariance matrix. The correlation pattern is then modified: in fit \#janw-7uncor systematics are assumed to be uncorrelated across rapidity bins, and in fit \#janw-7pcor systematics are partially decorrelated, 
Table 5 Same as Table 3 for fits performed with alternative choices of central scale or alternative decorrelation models. Now only $\chi^{2}$ values for jet data are shown. Results for the fits with default settings $\#$ j7, $\# \mathrm{j} 7 \mathrm{n}$ and \#janw, already shown in Table 3 are included for ease of ref- erence. Note that for the fits with alternative decorrelation models for the ATLAS $7 \mathrm{TeV}$ data (\#janw-7cor, \#janw-7dec and \#janw-7pcor) the number of ATLAS $7 \mathrm{TeV}$ data is $n_{\text {dat }}=140$ instead of $n_{\text {dat }}=30$ as for all other fits

\begin{tabular}{|c|c|c|c|c|c|c|c|c|c|c|c|}
\hline Dataset & $n_{\text {dat }}$ & j7 & j7-pt & $\mathrm{j} 7 \mathrm{n}$ & j7n-pt & janw & janw-7cor & janw-7dec & janw-7pcor & janw-8dec & janw-8pcor \\
\hline ATLAS jets $7 \mathrm{TeV}$ & $31(140)$ & 1.12 & 1.13 & 1.13 & 1.13 & 1.59 & 2.44 & 1.22 & 1.22 & 1.59 & 1.61 \\
\hline ATLAS jets $8 \mathrm{TeV}$ & 171 & [3.55] & [3.93] & [4.76] & [4.99] & 3.22 & 3.16 & 3.19 & 3.20 & 0.83 & 0.98 \\
\hline CMS jets $7 \mathrm{TeV}$ & 133 & 0.71 & 0.91 & 0.95 & 0.94 & 1.09 & 1.04 & 1.04 & 1.04 & 1.12 & 1.12 \\
\hline CMS jets $8 \mathrm{TeV}$ & 185 & [1.24] & {$[1.16]$} & [1.47] & {$[1.81]$} & 1.25 & 1.52 & 1.53 & 1.54 & 1.42 & 1.42 \\
\hline ATLAS dijets $7 \mathrm{TeV}$ & 90 & {$[1.77]$} & [1.98] & {$[2.46]$} & {$[2.55]$} & [1.95] & [1.86] & [1.86] & [1.88] & [1.98] & [1.98] \\
\hline CMS dijets $7 \mathrm{TeV}$ & 54 & {$[2.43]$} & {$[2.52]$} & {$[2.50]$} & {$[2.57]$} & {$[2.08]$} & [1.90] & [1.96] & [1.89] & [2.19] & {$[2.17]$} \\
\hline CMS dijets $8 \mathrm{TeV}$ & 122 & {$[6.70]$} & [7.48] & [3.53] & [3.89] & {$[2.21]$} & [1.95] & {$[2.46]$} & {$[2.47]$} & [2.96] & [3.04] \\
\hline
\end{tabular}
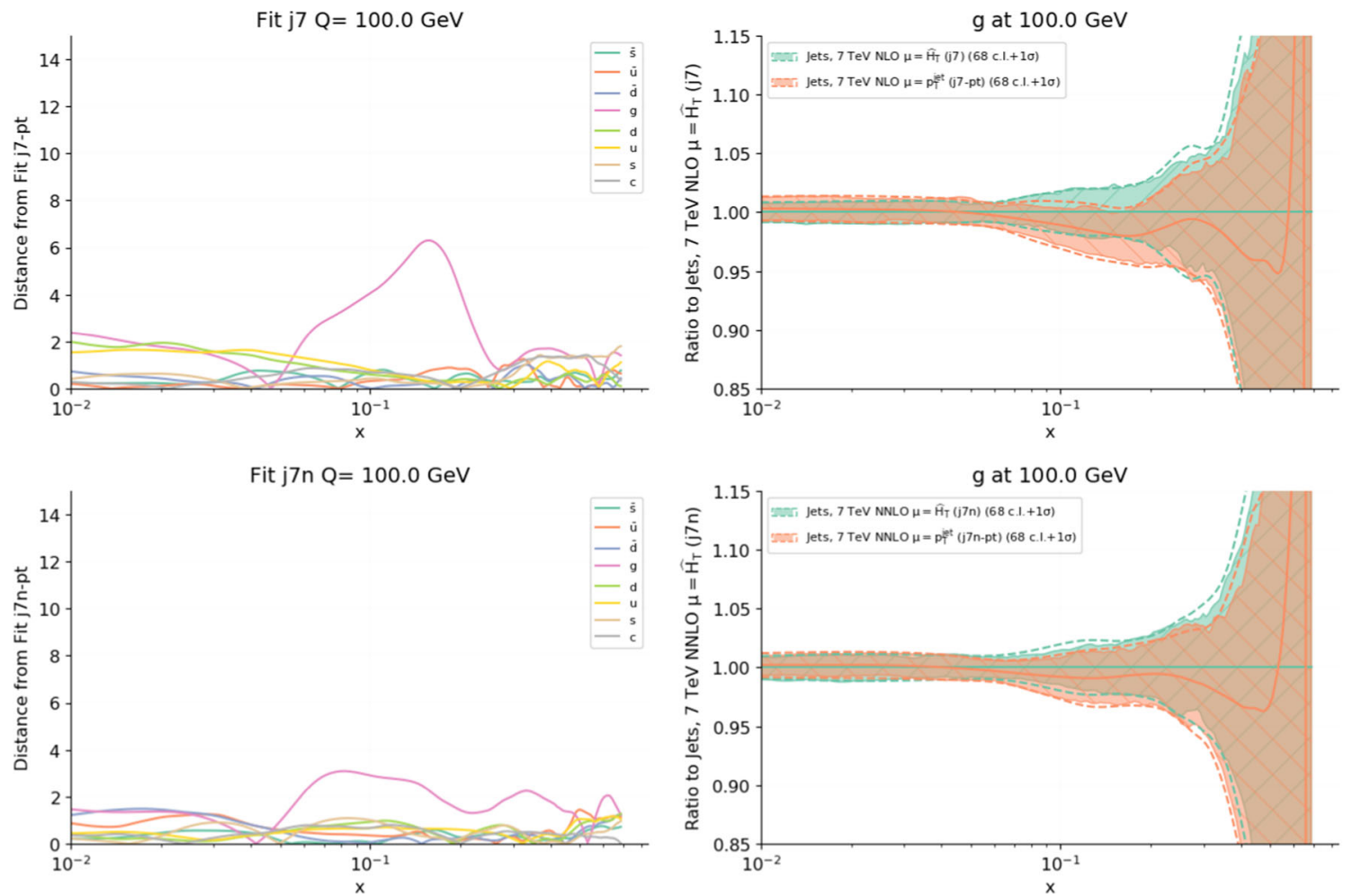

Fig. 9 Same as Fig. 6, but now comparing fits to the $7 \mathrm{TeV}$ data with the choices of central renormalization and factorization scale scale $\mu=\widehat{H}_{T}$ (as shown in Fig. 8, top) and $\mu=p_{T}^{\text {jet }}$ at NLO (fits \#j7 and \#j7-pt) and NNLO (fits \#j7n and \#j7n-pt). The gluon is shown as ratio to the fits with $\mu=\widehat{H}_{T}$

following the prescription suggested in Ref. [24]. Concerning the $8 \mathrm{TeV}$ data, we start with the default fit (\#janw), and we obtain from it two variants by modifying, as suggested in Ref. [15], the treatment of three (out of 659) correlated systematic uncertainties, related to the jet energy scale, specifically to the flavour response, the fragmentation and the pile-up. In fit \#janw-8dec these three uncertainties are completely decorrelated; in fit \#janw-8pcor they are partly decorrelated by splitting each uncertainty into three components and decorrelating one of them (see Table 6 or Ref. [15]).
The fit with only the central rapidity bin of the ATLAS $7 \mathrm{TeV}$ data (\#janw), and the fit in which all ATLAS data are included, with fully correlated systematics (\#janw-cor), are compared in Fig. 10, where we show distances between the two sets of PDFs, and we compare directly the gluon PDFs, shown as a ratio to the default optimal fit. It is clear that all PDFs including the gluon are essentially unchanged. On the other hand, the $\chi^{2}$ now increases very substantially. However, the $\chi^{2}$ to all other jet and dijet data (both fitted and not fitted) is essentially unchanged, consistently with the fact that the gluon is very stable. In short, we confirm the 
previous conclusion $[20,45]$ that including all rapidity bins of the ATLAS $7 \mathrm{TeV}$ data has almost no impact on the PDFs, despite the considerable deterioration of the $\chi^{2}$ for this data (Fig. 11).

Inspection of the $\chi^{2}$ values from Table 5 further reveals that as soon as systematics are decorrelated the $\chi^{2}$ value improves considerably. However, once again the $\chi^{2}$ to all other jet and dijet data changes very little. Hence once again we conclude that the PDFs are stable upon decorrelation. In fact, all $\chi^{2}$ values remain essentially the same regardless of whether correlations are completely removed, or only partially removed following the suggestion of Ref. [24], thereby validating the conclusion that these correlations are the problematic ones. The stability of PDFs in general and the gluon in particular upon decorrelation is confirmed by a direct comparison, shown in Fig. 10. Distances between all PDFs before and after decorrelation is seen to be compatible with statistical fluctuations.

Turning now to the $8 \mathrm{TeV}$ data, a similar pattern is found. Namely, upon decorrelation the $\chi^{2}$ for the ATLAS data improves considerably, but $\chi^{2}$ values for all other datasets are almost unaffected, with very similar results obtained when fully or partially decorrelating the relevant sources of systematics following Ref. [15], thus validating the prescription of this reference. Also in this case, the stability of the PDFs is confirmed by direct comparison in Fig. 12.

In summary, firstly, we confirm the conclusion of Refs. [20, 45] that inclusion of all of the ATLAS $7 \mathrm{TeV}$ jet data with full correlations has a significant impact on the fit quality but not on the PDFs. Furthermore, we confirm that the correlation model suggested in Ref. [24] leads to a good description of this data, without any significant change in the PDFs when the decorrelation is performed. And finally, we find that the ATLAS $8 \mathrm{TeV}$ data behave in a very similar way, and in particular that the correlation model suggested in Ref. [15] leads to good fit quality without significant change in PDFs.

\subsection{PDF fits with dijet data}

We now turn to PDF fits in which dijet data rather that singleinclusive jet data are included. Also in this case, we first discuss the impact and compatibility of these data, and then the perturbative stability of results.

\subsubsection{Impact and consistency of datasets}

We assess the impact of dijet data on PDFs by comparing fits with optimal settings, i.e. with NNLO QCD theory, and EW corrections included (for jets only), and either the full dataset (\#danw), or the $7 \mathrm{TeV}$ (\#d7nw) or $8 \mathrm{TeV}$ (\#d8nw) data included in turn.

We start by comparing to the baseline \#bn, with no jet data, fit \#danw in which all dijet data are included; PDFs are compared in Fig. 13. From Table 4, we see that individual dijet datasets are overall fairly well described (the $\chi^{2}$ per datapoint is around 1.5 for each of them). Inclusion of the dijet datasets in the baseline leads to an improved description of single-inclusive jet data, just like (see Sect. 4.2) inclusion of single-inclusive jet data leads to an improved description of dijets. This confirms consistency of the single-inclusive and dijet data. Unlike in the case of single-inclusive jet data, no tension is observed between dijet data and the rest of the global dataset (specifically top rapidity distributions), whose $\chi^{2}$ is left almost unchanged.

As in the case of single-inclusive jets, only the gluon PDF is affected by the inclusion of dijet data, with the strongest impact observed in the regions $x \simeq 0.01$ and $0.06 \lesssim x \lesssim 0.4$ (see Fig. 13). In the former region the gluon is suppressed by about $2 \%$, corresponding to a shift in central value by about one sigma; in the latter it is enhanced by up to $10 \%$ around $x \sim 0.3$, corresponding to a shift by about one and a half sigma, hence outside the error band of the baseline. These shifts are qualitatively similar to those observed upon inclusion of the single-inclusive jet data, but somewhat more pronounced and in a somewhat wider kinematic region.

We then turn to the assessment of the relative impact of different datasets, by comparing to the baseline (\#bn) the fits in which only $7 \mathrm{TeV}$ (\#d7nw) or only $8 \mathrm{TeV}$ (\#d8nw) dijet data are included, see Fig. 14. From Table 4, we see that the fit quality is equally good for $7 \mathrm{TeV}$ or $8 \mathrm{TeV}$ data, however the fit to the $8 \mathrm{TeV}$ dijet data is closer to the fit in which all dijet data are included, in that it leads to a similar description of all of the jet and dijet data, including those that are not included in either fit. Such a description is better in both fits than in the fit to the $7 \mathrm{TeV}$ dijet datasets only, and is accompanied by a similar change in the description of the ATLAS top pair differential rapidity distributions. This suggests that among the dijet data, the $8 \mathrm{TeV}$ data provide the dominant contribution.

The relative impact of the $7 \mathrm{TeV}$ and $8 \mathrm{TeV}$ data on the gluon central values and uncertainty can be directly inferred from Fig. 14. The impact of the two datasets on the gluon central value is qualitatively the same, and thus also the same as that of the full dijet dataset, but with the $8 \mathrm{TeV}$ data having a stronger impact, almost equivalent to the impact of the full dataset. The reduction in uncertainty in comparison to the baseline due to either dataset is almost the same, with a slightly stronger reduction observed for the $7 \mathrm{TeV}$ data, by about $3-4 \%$ to $3 \%$ at $x \simeq 0.2$. Consequently, the gluon PDF determined when including all of the dijet data is very close to that found when including only the $8 \mathrm{TeV}$ data, thus confirming that the $8 \mathrm{TeV}$ data have a dominant impact on the gluon central value. 

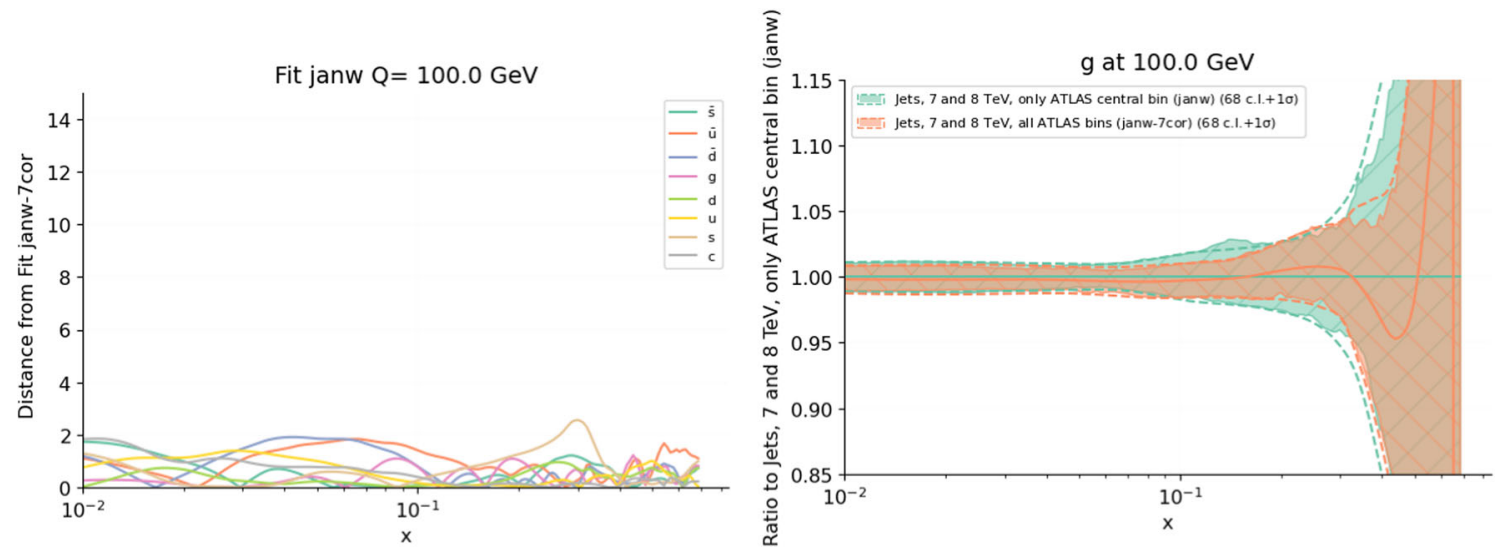

Fig. 10 Same as Fig. 6, but now comparing the default most accurate fit to single-inclusive jet data (all datasets, NNLO QCD+EW, fit \#janw), in which only the central rapidity bin of the ATLAS $7 \mathrm{TeV}$ data

is included, to a fit in which all rapidity bins are included (\#janw-7cor). The gluon is shown as ratio to the former fit
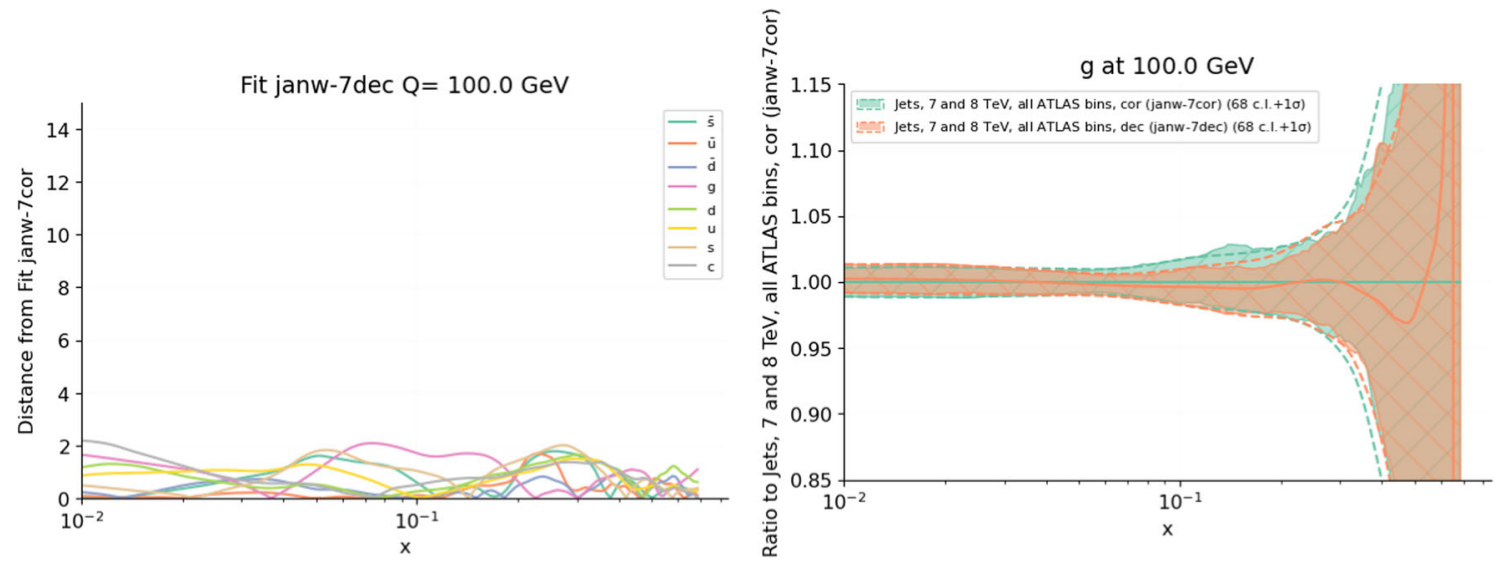

Fig. 11 Same as Fig. 6, but now comparing the fits to single-inclusive jet data (all datasets, NNLO QCD+EW), in which all rapidity bins of the ATLAS $7 \mathrm{TeV}$ data are included, either with (fit \#janw-7cor) or with-

out (fit \#janw-7dec) experimental correlations on selected systematic uncertainties. The gluon is shown as ratio to the former fit
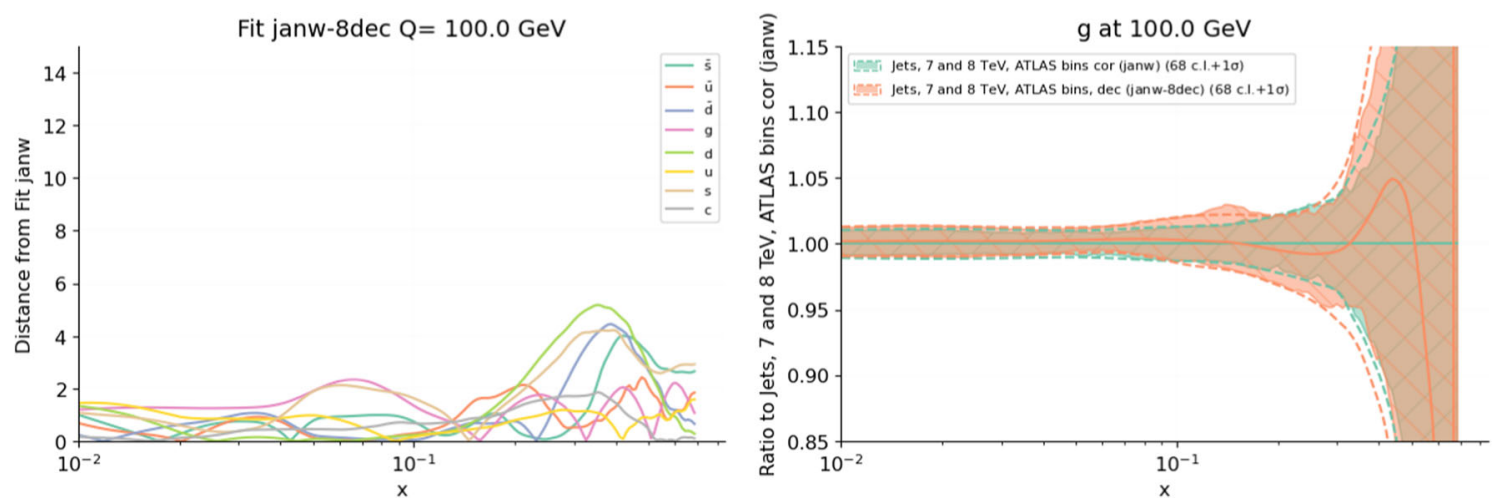

Fig. 12 Same as Fig. 6, but now comparing the default most accurate fit to single-inclusive jet data (all datasets, NNLO QCD+EW, fit \#janw), to a fit in which selected systematic uncertainties are decorrelated in the ATLAS $8 \mathrm{TeV}$ data (fit \#janw-8dec). The gluon is shown as ratio to the former fit 

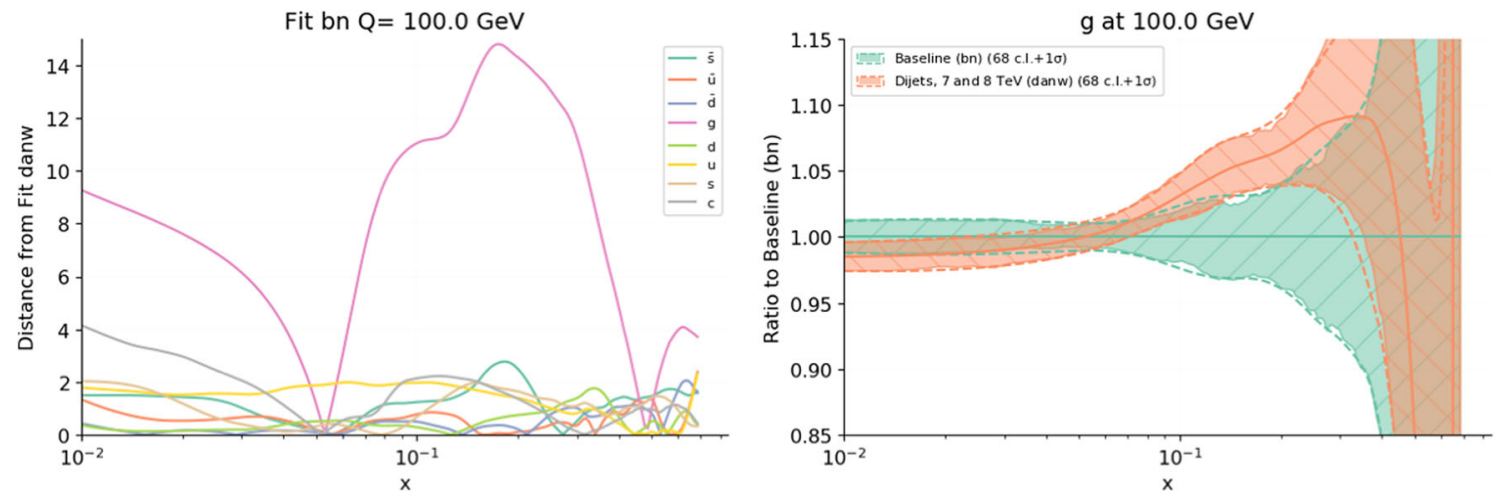

Fig. 13 Same as Fig. 6, but now for dijets
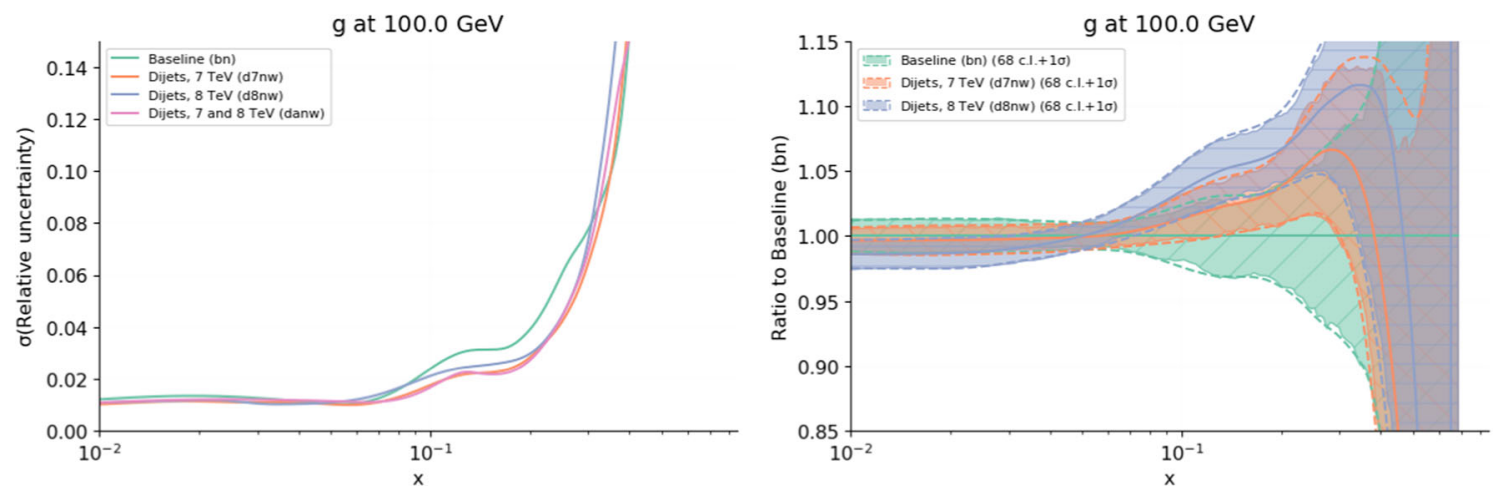

Fig. 14 Same as Fig. 7, but now for dijets
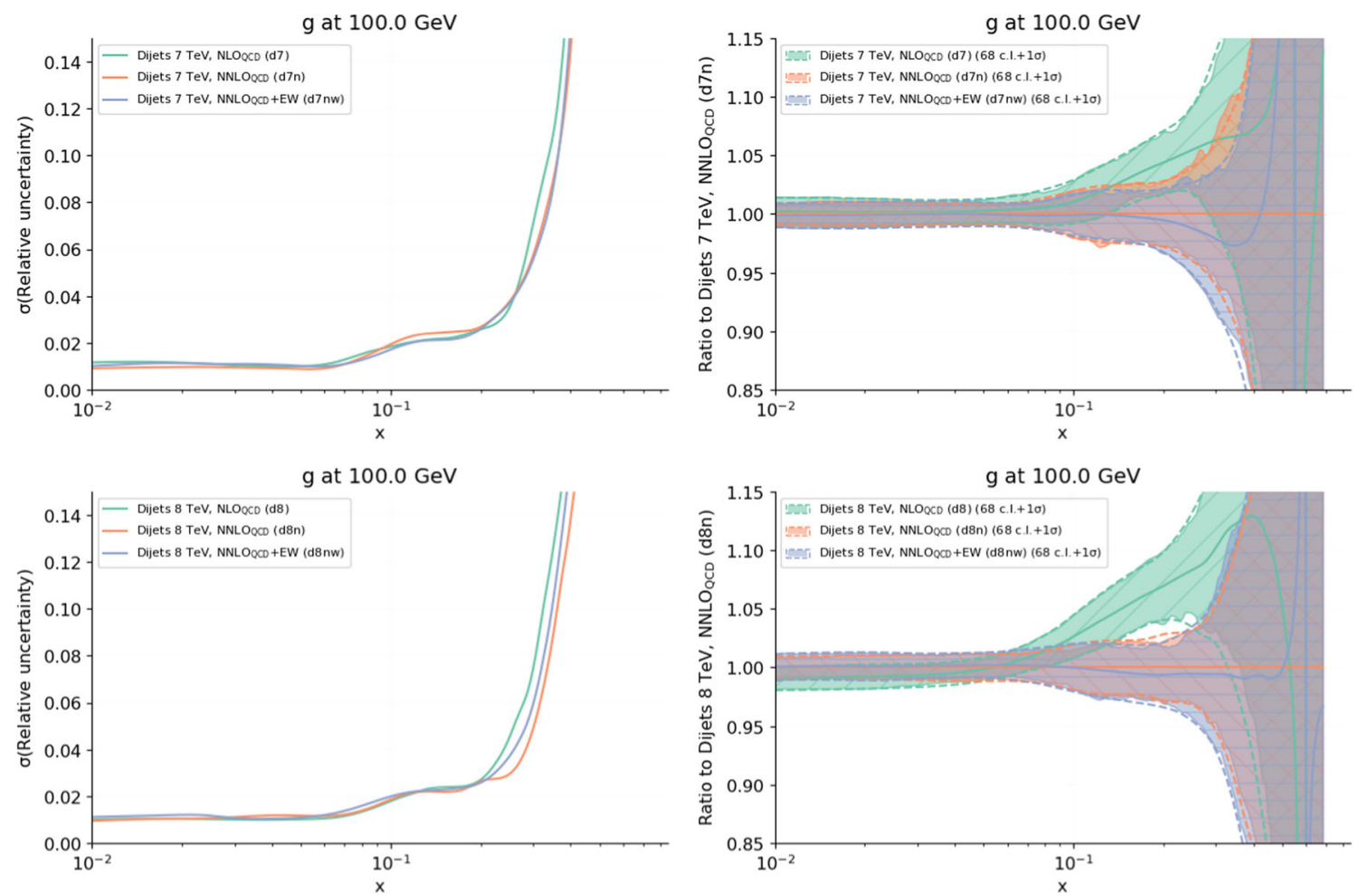

Fig. 15 Same as Fig. 8, but now for dijets 


\subsubsection{Impact of higher order QCD and EW corrections}

As for single-inclusive jets, we assess the perturbative stability of fits with dijet data by comparing fits at NLO, NNLO and with EW corrections, separately for the $7 \mathrm{TeV}$ and $8 \mathrm{TeV}$ data, i.e., respectively, fits \#d7, \#d7n, \#d7nw; and \#d8, \#d8n, \#d8nw. The gluon PDFs for these fits are compared in Fig. 15.

The figure shows that the perturbative behavior of the gluon upon inclusion of the dijet data is very similar to what observed when including single-inclusive jets. Namely, at NLO the gluon is distorted in the region $0.1 \lesssim x \lesssim 0.5$ in comparison to the NNLO results already shown in Fig. 14, with the effect more pronounced for the dominant and more precise $8 \mathrm{TeV}$ data, again providing evidence for large missing NNLO corrections. The effect of the EW corrections is even less marked than in the case of single-inclusive jets: in fact, their inclusion leaves the gluon PDF almost unchanged. For both datasets, inclusion of the NNLO corrections leads to a reduction in uncertainty, more marked for $8 \mathrm{TeV}$ data, while inclusion of the EW corrections has no clear effect; in fact, for the $8 \mathrm{TeV}$ data it leads to a slight increase of the uncertainty. As in the case of single-inclusive jets, we conclude that NNLO corrections have a strong impact by modifying the gluon shape and reducing its uncertainty, while EW corrections have essentially no impact.

Unlike in the case of single-inclusive jets, where the inclusion of NNLO corrections did not have a clear impact on fit quality, for dijets at NNLO there is a clear improvement in $\chi^{2}$ values (see Table 4). Specifically, when all dijet data are included at NLO, the $\chi^{2}$ of the global fit deteriorates significantly in comparison to the baseline, with the largest effect seen in data which are most sensitive to the gluon, such as the $Z p_{T}$ distribution and the top rapidity distribution. This deterioration goes away upon inclusion of NNLO corrections. Indeed, when NNLO corrections are included, the quality of the global fit including dijets improves considerably, corresponding now to a fit quality which is essentially the same for the fits with or without the dijet data. Accordingly, the fit quality to the dijet data is significantly better at NNLO than at NLO. The effect is driven by the more precise $8 \mathrm{TeV}$ data. Indeed, the same pattern is observed when only $8 \mathrm{TeV}$ data are included, while with $7 \mathrm{TeV}$ data only fit quality to the dijet data at NLO and NNLO is essentially the same, and so is the fit quality with or without dijet data.

This means that inclusion of NNLO corrections is crucial in order to ensure compatibility of the dijet data with the rest of the global dataset. Interestingly, when fitting dijet data no clear improvement in the fit quality of single-inclusive jet data (not fitted) is seen when going from NLO to NNLO. Inclusion of EW corrections has no significant effect on fit quality.

We conclude that for dijets NNLO corrections have a significant impact on both fit quality, the central value of the gluon PDF and its uncertainty, with a clear pattern of improvement when going from NLO to NNLO.

\subsection{Single-inclusive jets vs. dijets: a comparative assessment}

Having assessed the impact on PDFs of jet and dijet datasets separately, we now assess them comparatively, in terms of perturbative stability, fit quality, and impact on PDFs. Specifically, we compare directly PDFs obtained in fits to all singleinclusive (\#janw) and dijet (\#danw) datasets with the most accurate NNLO+EW theory and default settings in Figs. 16 and 17, where the baseline fit (with no jet data) and, in the latter case, the CT18 PDF fit [22] are also shown for reference. Also, in Figs. 18 and 19 we compare to a representative set of datapoints from each of the single-inclusive jet and dijet datasets predictions obtained using PDFs from the baseline fit, the fit with single-inclusive jets, and the fit with dijets. Predictions are shown as a ratio to the experimental data, which are shown either with full uncertainties, or with uncorrelated uncertainties only, with the correlated uncertainties kept into account as a shift of the datapoint (see e.g. Eqs. (85-86) of Ref. [5]).

Based on the $\chi^{2}$ values from Tables 3 and 4 and the PDF comparisons in Figs. 16, 17, 18, 19, our conclusions are the following.

1. Concerning the relative impact on PDFs of singleinclusive jets and dijets:

(a) The effect on PDFs of the inclusion of jet and dijet data in the NNPDF3.1 global dataset is qualitatively the same. Namely, they only affect the gluon, by leading to an enhancement of its central value in the region $0.1 \lesssim x \lesssim 0.4$, accompanied by a suppression in the region $0.01 \lesssim x \lesssim 0.1$. The suppression is by about $1 \%$, while the enhancement at the peak, localized at $x \simeq 0.3$ is by about $2.5 \%$ for single-inclusive jets, but stronger, by about $7.5 \%$ for dijets. An enhanced gluon is also present in the CT18 PDF determination, which, as mentioned, includes the $8 \mathrm{TeV}$ CMS singleinclusive jet data, and whose gluon PDF is consistent with our result within its rather larger uncertainty.

(b) The inclusion of either single-inclusive or dijets leads to a reduction in the gluon uncertainty, with a somewhat stronger reduction observed for single-inclusive jets. It should be noted in this respect that for the most accurate $8 \mathrm{TeV}$ dijet dataset, which as shown in Sects. 4.2.1-4.3.1 is mostly responsible for the shift in central value (though not on the uncertainty), only CMS data are currently available. The constraining power of the dijet dataset is consequently at 


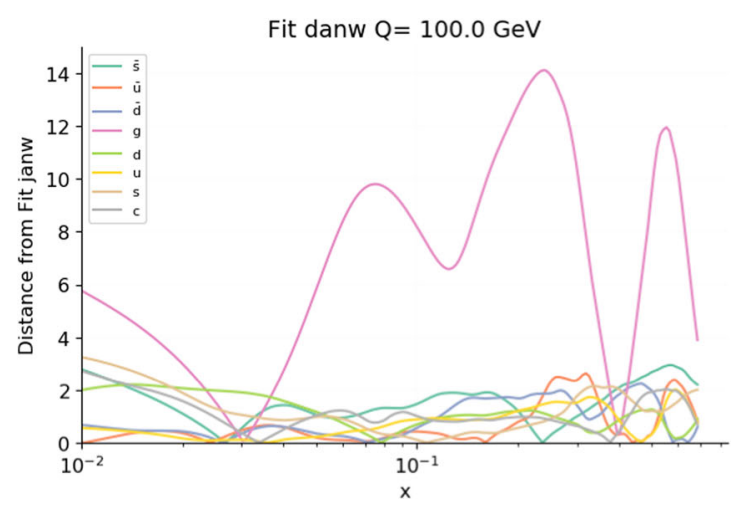

Fig. 16 Same as Fig. 6, but now comparing the fits with all singleinclusive jet data (\#janw), and that with all dijet data (\#danw) and highest theory accuracy (NNLO QCD+ EW) and default settings. In the

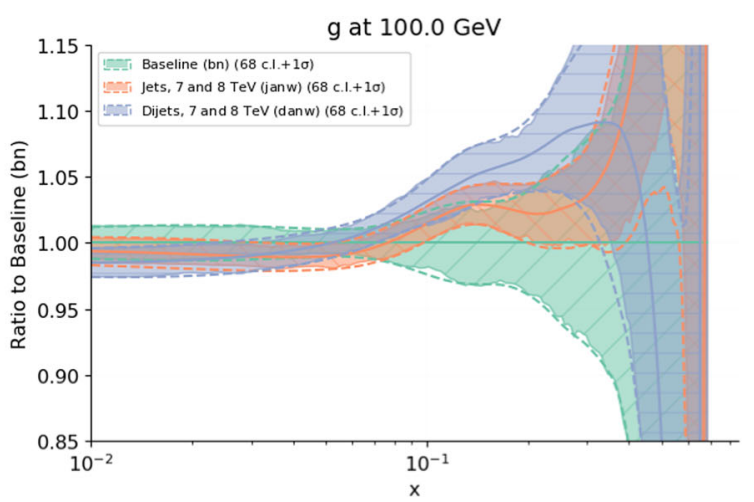

gluon comparison (right) results are displayed as a ratio to the baseline with no jet data included (also shown for reference)
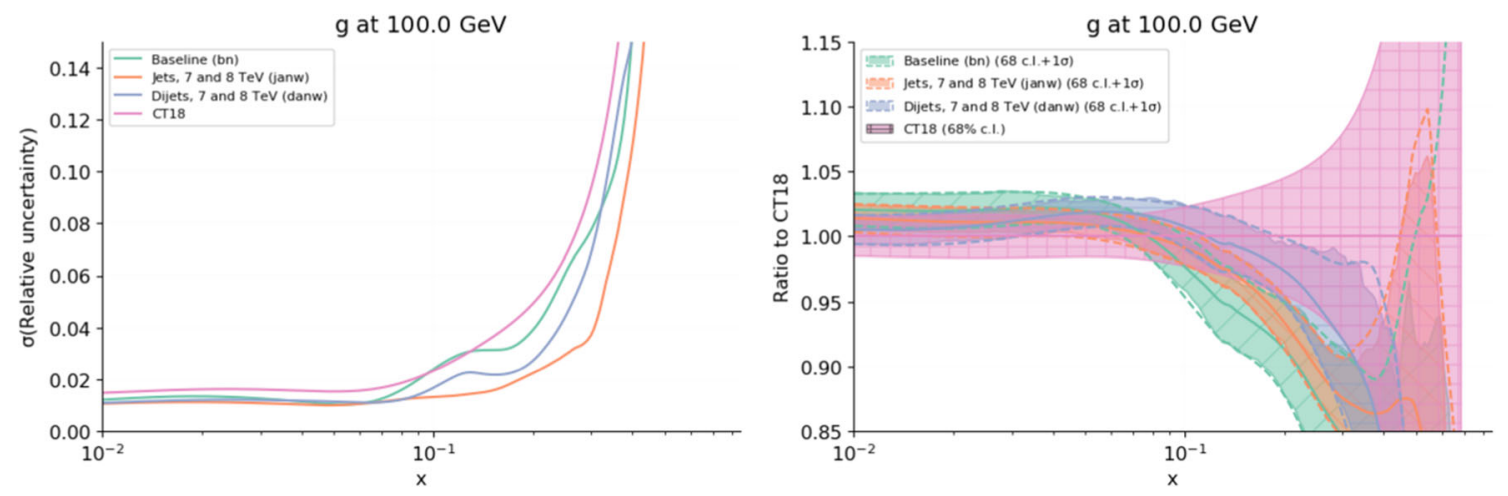

Fig. 17 Same as Fig. 7, but now comparing the baseline (\#bn) to the fits with all single-inclusive jet (\#janw) and dijet data (\#danw) of Fig. 16. All results are shown as a ratio to the CT18 fit (also shown for reference)

present more limited than that of the single-inclusive jet dataset.

(c) The inclusion of single-inclusive jet or dijet data does not lead to a deterioration in the description of the rest of the data in comparison to the baseline fit: almost all $\chi^{2}$ values for other datasets are unchanged. This shows that the single-inclusive and dijet data are not only consistent with each other, but also with the rest of the global dataset, and their impact on the gluon central value, accompanied by a reduction in uncertainty, corresponds to a genuine addition of new information in the fit. Indeed, a comparative assessment of the impact of jet, $Z p_{T}$ and top production data on the gluon distribution in Ref. [45] showed good consistency, specifically wih the top data also leading to an enhancement of the gluon in the $x \gtrsim 0.1$ region. An exception is the ATLAS top rapidity distributions, which seem to be in tension with the ATLAS $8 \mathrm{TeV}$ single-inclusive jet data, as discussed in Sect. 4.2.1. The quality of the fit to this data also deteriorates, though by a smaller amount, when dijet data are fit- ted; note however that in this case the quality of the fit to CMS top rapidity data improves.

2. Concerning relative fit quality:

(a) The quality of the fit to single-inclusive jet data and dijet data when each of them is fitted is comparable, though somewhat better for dijets $\left(\chi^{2}=1.65 \mathrm{vs}\right.$. $\left.\chi^{2}=1.88\right)$. The quality of the fit to dijets when single inclusive jets are fitted and conversely are almost identical $\left(\chi^{2}=2.10\right.$ for dijets when fitting singleinclusive jets vs. $\chi^{2}=2.06$ for single-inclusive jets when fitting dijets), and only marginally worse than the quality of the fit to each dataset when it is fitted. This confirms the full consistency of the two datasets, with a marginal preference for dijets.

(b) The fit including dijet data is also somewhat more internally consistent than the fit including singleinclusive jet data. Indeed, the $\chi^{2}$ per datapoint of the global fit is closer to one (1.22 vs 1.28), and also, the $\chi^{2}$ for individual datasets is generally better. In particular, this happens for top production data, also sensitive to the large- $x$ gluon. It is unclear whether 
this is due to a greater theoretical accuracy of the NNLO dijet observable, or to better quality of the dijet data (specifically a better control of correlated systematics). However, the issue is phenomenologically immaterial, given that the shape and size of the data to theory ratio are qualitatively comparable for all of the jet and dijet data (including for the rapidity bins not displayed in Figs. 18 and 19), regardless of which dataset is actually fitted.

3. Concerning relative perturbative stability:

(a) When fitting the dijet data, fit quality to the fitted data improves significantly from NLO to NNLO $\left(\chi^{2}=2.44\right.$ at NLO vs. 1.65 at NNLO), but the fit quality to the single-inclusive jet data actually deteriorates from NLO to NNLO (from $\chi^{2}=1.54$ to 2.06). When fitting the single-inclusive jet data, the fit quality to the fitted data does not improve and actually deteriorates from NLO to NNLO (from $\chi^{2}=1.25$ to $\chi^{2}=1.88$ ) but, perhaps surprisingly, the fit quality to the dijet data, not fitted, does improve (from $\chi^{2}=3.29$ at NLO to the NNLO $\chi^{2}=2.10$ ). Whereas this shows a good theoretical consistency of the dijet data, it is unclear whether the lack of improvement of the single-inclusive jet data is due to a less stable perturbative behavior of the jet observable, or to issues with data.

(b) As already noted in Sect. 4.3.2, the fit quality to all other data included in the global datasets deteriorates at NLO when including jet data, with a greater deterioration seen in the case of dijets, and more moderate for single-inclusive jets: the total $\chi^{2}$ per datapoint for the global fit goes from $\chi^{2}=1.20$ of the baseline to 1.28 in the former case and 1.33 in the latter. At NNLO, when dijets are fitted the global fit quality significantly improves and becomes almost the same as that of the baseline $\left(\chi^{2}=1.22\right.$, in comparison to $\chi^{2}=1.18$ of the baseline) while for the fit to single-inclusive jets it does not improve. The greater deterioration of fit quality at NLO for dijets can be understood as a consequence of the fact, observed in point 1.a above, that dijets have a greater pull on the gluon: hence missing NNLO corrections lead to a stronger loss of accuracy. The lack of improvement in the description of single-inclusive jets shows again that this observable seems to be somewhat less wellbehaved, either for theoretical or experimental reasons.

We generally conclude that single-inclusive jets and dijets are mutually consistent and at NNLO consistent with the global dataset and have a similar impact on the gluon. The dijet observable has a better behaved perturbative behavior and a stronger pull on the gluon PDF and it appears to be marginally preferable, though it leads to a less pronounced decrease of the gluon uncertainty, possibly because ATLAS dijet measurements are not yet available at $8 \mathrm{TeV}$, while single-inclusive jet measurements are available both from ATLAS and CMS.

\section{Summary and outlook}

We have presented an extensive phenomenological investigation of inclusive jet production at the LHC, exploiting recent theory calculations, in particular of NNLO QCD corrections, and studying for the first time in a systematic and comparative way the inclusive dijet observable, along with the single-inclusive jet observable which is routinely used for PDF determination.

We have found full consistency between the constraints imposed on parton distributions, specifically the gluon, by single-inclusive jets and dijets, thus conclusively establishing the viability of the dijet observable for precision QCD phenomenology and PDF determination, as originally suggested 25 years ago [9]. We have also validated the conclusions of previous theoretical studies [1-3,10-12]. Specifically we have shown that NNLO corrections are crucial in order to ensure compatibility of the jet observables with the rest of the global dataset, and also that while at NLO the choice of central scale makes a significant impact (with the scale choice $\widehat{H}_{T}$ for single-inclusive jets better behaved at NLO), at NNLO perturbative stability appears to be achieved.

In a comparative assessment of single-inclusive jets vs. dijets, we have found that the dijet observable has a more marked impact on the gluon central value. Also, phenomenologically it displays a better-behaved perturbative behavior, with a clear improvement of data-theory agreement as the perturbative order of the theory prediction increases. However, the single-inclusive jet observable leads to a more significant reduction of the gluon uncertainty. Either, or both, of the latter observations could be due to theoretical reasons but also to the nature of the current data. Specifically, there are indications that some of the single-inclusive datasets are in tension with the rest of the global datasets, which could explain the less clear perturbative behavior of fits including this observable. Also, the current dijet dataset is more limited than the single-inclusive dataset, thus possibly explaining the more limited effect of dijets on the gluon uncertainty.

One of our main results is that the more recent $8 \mathrm{TeV}$ data generally have a rather more significant impact than previous $7 \mathrm{TeV}$ data; interestingly, the dijet $8 \mathrm{TeV} \mathrm{CMS}$ data are tripledifferential, and this may enhance their impact on PDF determination. We accordingly expect that the availability of more precise data, possibly also for a greater variety of kinematic observables, including more differential measurements, from 

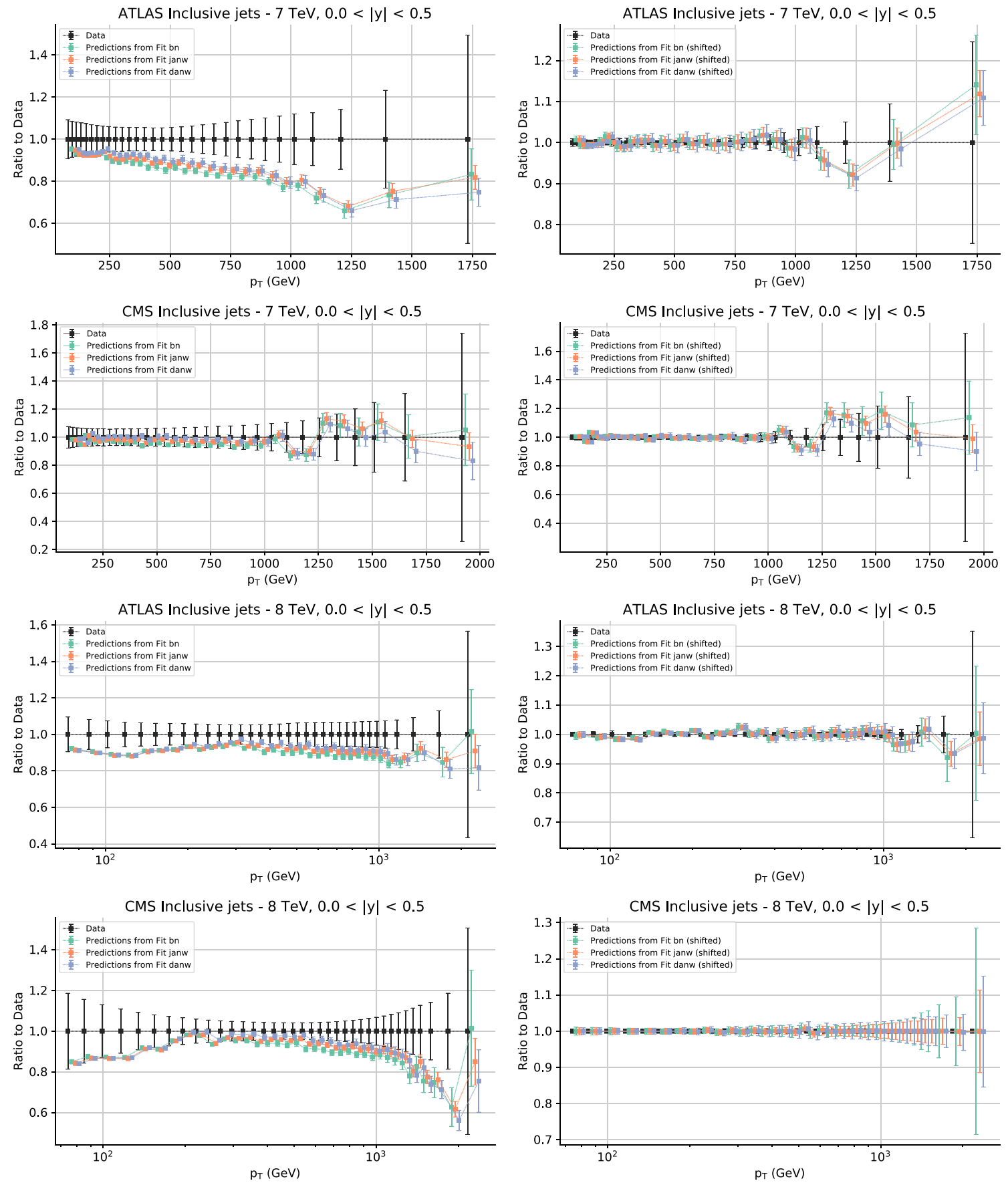

Fig. 18 The theory to data ratio for a representative set of points from each single-inclusive jet dataset introduced in Sect. 2. Specifically, we show the central rapidity bins for all the ATLAS and CMS jet datasets at 7 and $8 \mathrm{TeV}$. Theoretical predictions are computed from fits \#bn, \#janw

and \#danw with corresponding theoretical accuracy, and shown as a ratio to the experimental data. For the data, either the full uncertainty is shown (left) or only the uncorrelated uncertainty, with the correlated uncertainty kept into account as a shift of the datapoint (right) 

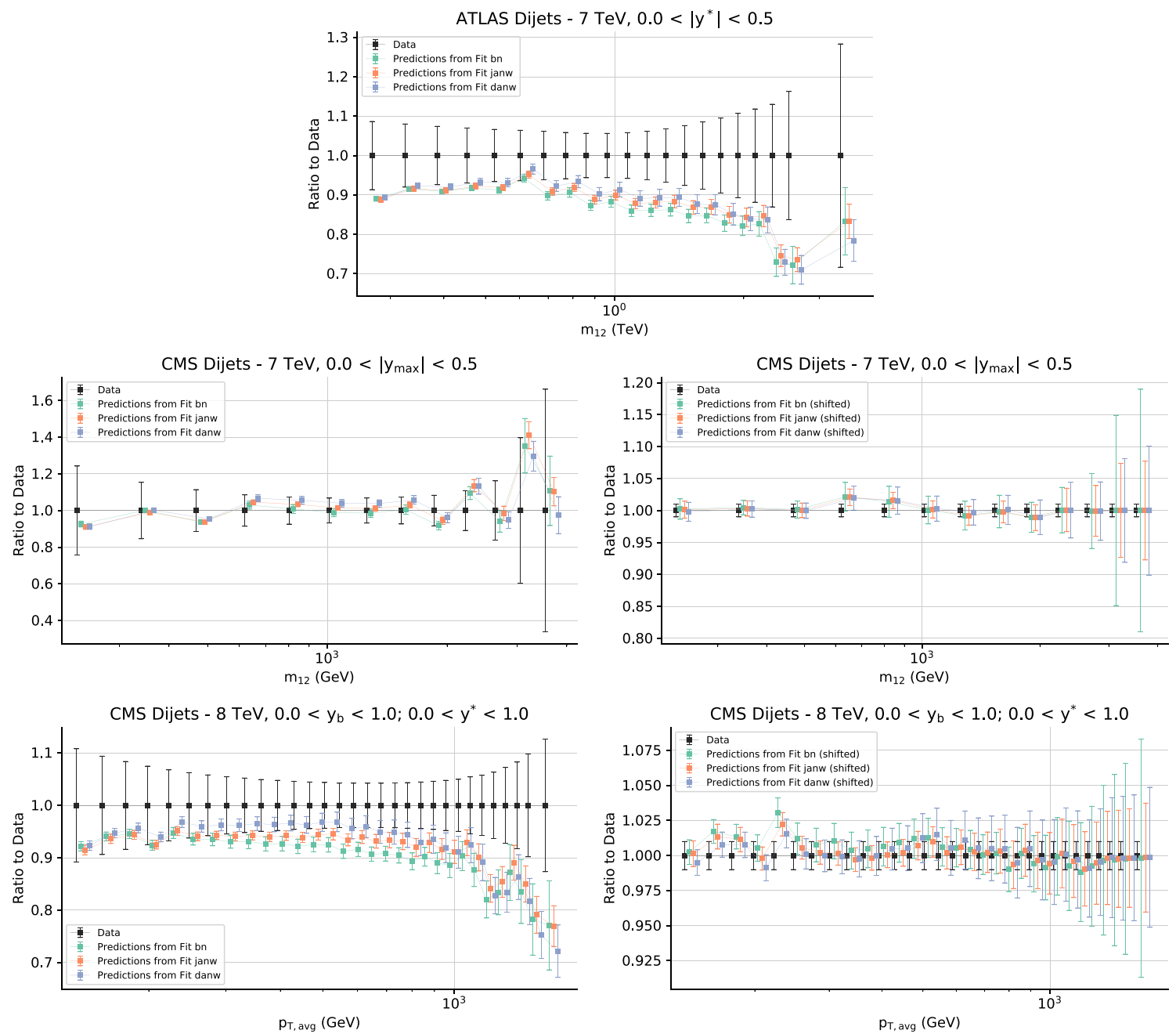

Fig. 19 Same as Fig. 18, but for dijets. For the ATLAS data only the unshifted data are shown because the breakdown of the statistical covariance matrix into fully uncorrelated components is not available

LHC Run-II $13 \mathrm{TeV}$ data, and then from data coming from future LHC Run-III and HL-LHC [102] runs will settle these issues and lead to a clear and consistent theoretical picture. Eventually, the availability of correlations between singleinclusive jet and dijets data will allow for their simultaneous inclusion in a PDF determination, thereby allowing for maximal use of the available information. Indeed, we expect this to be a first step towards a widening of the set of jet observables used in precision PDF studies, which include not only multi-differential jet cross-sections [46], but also jet substructure observables, whose study has undergone rapid progress recently [103]. The inclusion of dijet data in the forthcoming NNPDF4.0 global PDF analysis will be a first step in this direction.

Acknowledgements S. F. is supported by the European Research Council under the European Union's Horizon 2020 research and innovation Programme (Grant agreement ERC-AdG-740006). T. G. is supported by The Scottish Funding Council, Grant H14027. E. R. N. is supported by the European Commission through the Marie SkłodowskaCurie Action ParDHonS FFs.TMDs (Grant number 752748). J. P. acknowledges the financial support by ERC2018-ADG-835105 YoctoLHC, Fundação para a Ciencia e Tecnologia (FCT, Portugal) through the contract UIDP/50007/2020 and by FCT under project CERN/FIS-PAR/0024/2019, and COST Action CA16201 PARTICLEFACE. J. R. is supported by an European Research Council Starting Grant "PDF4BSM" and by the Netherlands Organization for Scientific Research (NWO). This work was supported in part by the Swiss National Science Foundation (SNF) under contract 200020-175595.

Data Availability Statement This manuscript has associated data in a data repository. [Authors' comment: The data associated to this work can be obtained from http://nnpdf.mi.infn.it/nnpdf3-1jets/.]

Open Access This article is licensed under a Creative Commons Attribution 4.0 International License, which permits use, sharing, adaptation, distribution and reproduction in any medium or format, as long as you give appropriate credit to the original author(s) and the source, provide a link to the Creative Commons licence, and indicate if changes were made. The images or other third party material in this article are included in the article's Creative Commons licence, unless indicated otherwise in a credit line to the material. If material is not 
included in the article's Creative Commons licence and your intended use is not permitted by statutory regulation or exceeds the permitted use, you will need to obtain permission directly from the copyright holder. To view a copy of this licence, visit http://creativecomm ons.org/licenses/by/4.0/.

Funded by SCOAP ${ }^{3}$.

\section{References}

1. A. Gehrmann-De Ridder, T. Gehrmann, E.W.N. Glover, J. Pires, Second order QCD corrections to jet production at hadron colliders: the all-gluon contribution. Phys. Rev. Lett. 110, 162003 (2013). arXiv:1301.7310

2. J. Currie, A. Gehrmann-De Ridder, E. Glover, J. Pires, NNLO QCD corrections to jet production at hadron colliders from gluon scattering. JHEP 1401, 110 (2014). arXiv: 1310.3993

3. J. Currie, E.W.N. Glover, J. Pires, NNLO QCD predictions for single jet inclusive production at the LHC. Phys. Rev. Lett. 118(7), 072002 (2017). arXiv:1611.01460

4. M. Czakon, A. van Hameren, A. Mitov, R. Poncelet, Single-jet inclusive rates with exact color at $\mathcal{O}\left(\alpha_{s}^{4}\right)$. JHEP 10, 262 (2019). arXiv: 1907.12911

5. J. Gao, L. Harland-Lang, J. Rojo, The structure of the proton in the LHC precision era. Phys. Rep. 742, 1-121 (2018). arXiv: 1709.04922

6. A.D. Martin, R.G. Roberts, W.J. Stirling, Structure function analysis and psi, Jet, W, Z production: pinning down the gluon. Phys. Rev. D 37, 1161 (1988)

7. F. Aversa, P. Chiappetta, M. Greco, J.P. Guillet, Higher order corrections to QCD jets. Phys. Lett. B 210, 225 (1988)

8. S.D. Ellis, Z. Kunszt, D.E. Soper, The one jet inclusive crosssection at order $\alpha_{s}^{3}$ : gluons only. Phys. Rev. Lett. 62, 726 (1989)

9. W.T. Giele, E.W.N. Glover, D.A. Kosower, The inclusive two jet triply differential cross-section. Phys. Rev. D 52, 1486-1499 (1995). arXiv:hep-ph/9412338

10. J. Currie, E.W.N. Glover, A. Gehrmann-De Ridder, T. Gehrmann, A. Huss, J. Pires, Single jet inclusive production for the individual jet $p_{T}$ scale choice at the LHC. In: 23rd Cracow Epiphany Conference on Particle Theory Meets the First Data from LHC Run 2 Cracow, Poland, January 9-12, 2017 (2017). arXiv:1704.00923

11. J. Currie, A. Gehrmann-De Ridder, T. Gehrmann, E.W.N. Glover, A. Huss, J. Pires, Infrared sensitivity of single jet inclusive production at hadron colliders. JHEP 10, 155 (2018). arXiv: 1807.03692

12. M. Cacciari, S. Forte, D. Napoletano, G. Soyez, G. Stagnitto, Single-jet inclusive cross section and its definition. Phys. Rev. D 100(11), 114015 (2019). arXiv:1906.11850

13. M. Dasgupta, F.A. Dreyer, G.P. Salam, G. Soyez, Inclusive jet spectrum for small-radius jets. JHEP 06, 057 (2016). arXiv: 1602.01110

14. ATLAS Collaboration, G. Aad et al., Measurement of the inclusive jet cross-section in proton-proton collisions at $\sqrt{s}=7 \mathrm{TeV}$ using $4.5 \mathrm{fb}^{-1}$ of data with the ATLAS detector. JHEP 02, 153 (2015). arXiv: 1410.8857

15. ATLAS Collaboration, M. Aaboud et al., Measurement of the inclusive jet cross-sections in proton-proton collisions at $\sqrt{s}=8 \mathrm{TeV}$ with the ATLAS detector. JHEP 09, 020 (2017). arXiv: 1706.03192

16. CMS. Collaboration, S. Chatrchyan et al., Measurements of differential jet cross sections in proton-proton collisions at $\sqrt{s}=7$ TeV with the CMS detector. Phys. Rev. D 87, 112002 (2013). arXiv: 1212.6660

17. CMS Collaboration, V. Khachatryan et al., Measurement and QCD analysis of double-differential inclusive jet cross sections in pp collisions at $\sqrt{s}=8 \mathrm{TeV}$ and cross section ratios to 2.76 and 7 TeV. JHEP 03, 156 (2017). arXiv: 1609.05331

18. ATLAS Collaboration Collaboration, G. Aad et al., Measurement of dijet cross sections in $p p$ collisions at $7 \mathrm{TeV}$ centre-ofmass energy using the ATLAS detector. JHEP 1405, 059 (2014). arXiv: 1312.3524

19. CMS Collaboration, A.M. Sirunyan et al., Measurement of the triple-differential dijet cross section in proton-proton collisions at $\sqrt{s}=8 \mathrm{TeV}$ and constraints on parton distribution functions. Eur. Phys. J. C77(11), 746 (2017). arXiv:1705.02628

20. NNPDF Collaboration, R.D. Ball et al., Parton distributions from high-precision collider data. Eur. Phys. J. C 77(10), 663 (2017). arXiv: 1706.00428

21. L.A. Harland-Lang, A.D. Martin, P. Motylinski, R.S. Thorne, Parton distributions in the LHC era: MMHT 2014 PDFs. Eur. Phys. J. C 75, 204 (2015). arXiv:1412.3989

22. T.-J. Hou et al., New CTEQ global analysis of quantum chromodynamics with high-precision data from the LHC. arXiv:1912.10053

23. C.M.S. Collaboration, V. Khachatryan et al., Constraints on parton distribution functions and extraction of the strong coupling constant from the inclusive jet cross section in pp collisions at $\sqrt{s}=7$ TeV. Eur. Phys. J. C 75(6), 288 (2015). arXiv: 1410.6765

24. L.A. Harland-Lang, A.D. Martin, R.S. Thorne, The impact of LHC jet data on the MMHT PDF fit at NNLO. Eur. Phys. J. C 78(3), 248 (2018). arXiv:1711.05757

25. M. Cacciari, G.P. Salam, G. Soyez, The anti-k(t) jet clustering algorithm. JHEP 0804, 063 (2008). arXiv:0802.1189

26. ATLAS Collaboration, M. Aaboud et al., Measurement of inclusive jet and dijet cross-sections in proton-proton collisions at $\sqrt{s}=13 \mathrm{TeV}$ with the ATLAS detector. JHEP 05, 195 (2018). arXiv: 1711.02692

27. CMS Collaboration, V. Khachatryan et al., Measurement of the double-differential inclusive jet cross section in proton-proton collisions at $\sqrt{s}=13 \mathrm{TeV}$. Eur. Phys. J. C76(8), 451 (2016). arXiv: 1605.04436

28. CMS Collaboration, A.M. Sirunyan et al., Measurements of the differential jet cross section as a function of the jet mass in dijet events from proton-proton collisions at $\sqrt{s}=13 \mathrm{TeV}$. JHEP 11, 113 (2018). arXiv: 1807.05974

29. CMS Collaboration, A.M. Sirunyan et al., Dependence of inclusive jet production on the anti- $k_{\mathrm{T}}$ distance parameter in pp collisions at $\sqrt{s}=13 \mathrm{TeV}$. arXiv:2005.05159

30. ATLAS Collaboration, G. Aad et al., Measurement of the inclusive jet cross section in pp collisions at $\sqrt{s}=2.76 \mathrm{TeV}$ and comparison to the inclusive jet cross section at $\sqrt{s}=7 \mathrm{TeV}$ using the ATLAS detector. Eur. Phys. J. C 73, 2509 (2013). arXiv:1304.4739

31. CMS Collaboration, V. Khachatryan et al., Measurement of the inclusive jet cross section in pp collisions at $\sqrt{s}=2.76 \mathrm{TeV}$. Eur. Phys. J. C 76(5), 265 (2016). arXiv:1512.06212

32. CMS Collaboration, A.M. Sirunyan et al., Constraining gluon distributions in nuclei using dijets in proton-proton and protonlead collisions at $\sqrt{s_{\mathrm{NN}}}=5.02 \mathrm{TeV}$. Phys. Rev. Lett. 121(6), 062002 (2018). arXiv: 1805.04736

33. K.J. Eskola, P. Paakkinen, H. Paukkunen, Non-quadratic improved Hessian PDF reweighting and application to CMS dijet measurements at 5.02 TeV. Eur. Phys. J. C 79(6), 511 (2019). arXiv: 1903.09832

34. NNPDF Collaboration, R. Abdul Khalek, J.J. Ethier, J. Rojo, Nuclear parton distributions from lepton-nucleus scattering and the impact of an electron-ion collider. Eur. Phys. J. C 79(6), 471 (2019). arXiv: 1904.00018

35. ATLAS Collaboration, G. Aad et al., Measurement of three-jet production cross-sections in $p p$ collisions at $7 \mathrm{TeV}$ centre-ofmass energy using the ATLAS detector. Eur. Phys. J. C 75(5), 228 (2015). arXiv:1411.1855 
36. ATLAS Collaboration, G. Aad et al., Measurement of fourjet differential cross sections in $\sqrt{s}=8 \mathrm{TeV}$ proton-proton collisions using the ATLAS detector. JHEP 12, 105 (2015). arXiv: 1509.07335

37. C.M.S. Collaboration, V. Khachatryan et al., Measurement of the inclusive 3-jet production differential cross section in protonproton collisions at $7 \mathrm{TeV}$ and determination of the strong coupling constant in the TeV range. Eur. Phys. J. C 75, 186 (2015). arXiv: 1412.1633

38. ATLAS Collaboration, G. Aad et al., Measurement of inclusive jet and dijet production in pp collisions at $\sqrt{s}=7 \mathrm{TeV}$ using the ATLAS detector. Phys. Rev. D 86, 014022 (2012). arXiv: 1112.6297

39. CDF-Run II Collaboration, A. Abulencia et al., Measurement of the inclusive jet cross section using the $k_{\mathrm{T}}$ algorithm in $p \bar{p}$ collisions at $\sqrt{s}=1.96 \mathrm{TeV}$ with the CDF II detector. Phys. Rev. D 75, 092006 (2007). arXiv:hep-ex/0701051

40. NNPDF Collaboration, R.D. Ball, S. Carrazza, L. Del Debbio, S. Forte, Z. Kassabov, J. Rojo, E. Slade, M. Ubiali, Precision determination of the strong coupling constant within a global PDF analysis. Eur. Phys. J. C 78(5), 408 (2018). arXiv: 1802.03398

41. NNPDF Collaboration, V. Bertone, S. Carrazza, N.P. Hartland, J. Rojo, Illuminating the photon content of the proton within a global PDF analysis. Sci. Post Phys. 5(1), 008 (2018). arXiv: 1712.07053

42. R.D. Ball, V. Bertone, M. Bonvini, S. Marzani, J. Rojo, L. Rottoli, Parton distributions with small-x resummation: evidence for BFKL dynamics in HERA data. Eur. Phys. J. C 78(4), 321 (2018). arXiv: 1710.05935

43. NNPDF Collaboration, R. Abdul Khalek et al., Parton Distributions with theory uncertainties: general formalism and first phenomenological studies. Eur. Phys. J. C 79(11), 931 (2019). arXiv: 1906.10698

44. NNPDF Collaboration, R. Abdul Khalek et al., A first determination of parton distributions with theoretical uncertainties. Eur. Phys. J. C 79, 838 (2019). arXiv:1905.04311

45. E.R. Nocera, M. Ubiali, Constraining the gluon PDF at large $x$ with LHC data. PoS DIS2017, 008 (2018). arXiv:1709.09690

46. A. Gehrmann-De Ridder, T. Gehrmann, E.W.N. Glover, A. Huss, J. Pires, Triple differential Dijet cross section at the LHC. Phys. Rev. Lett. 123(10), 102001 (2019). arXiv:1905.09047

47. J. Currie, A. Gehrmann-De Ridder, T. Gehrmann, E.W.N. Glover, A. Huss, J. Pires, Precise predictions for Dijet production at the LHC. Phys. Rev. Lett. 119(15), 152001 (2017). arXiv:1705.10271

48. J. Currie, A. Gehrmann-De Ridder, T. Gehrmann, E. W. N. Glover, A. Huss, J. Pires, Jet cross sections at the LHC with NNLOJET. PoS LL2018, 001 (2018). arXiv:1807.06057

49. S. Dittmaier, A. Huss, C. Speckner, Weak radiative corrections to dijet production at hadron colliders. JHEP 1211, 095 (2012). arXiv: 1210.0438

50. Z. Nagy, Three jet cross-sections in hadron hadron collisions at next-to-leading order. Phys. Rev. Lett. 88, 122003 (2002). arXiv:hep-ph/0110315

51. fastNLO Collaboration, M. Wobisch, D. Britzger, T. Kluge, K. Rabbertz, F. Stober, Theory-data comparisons for jet measurements in hadron-induced processes. arXiv: 1109.1310

52. V. Bertone, S. Carrazza, N.P. Hartland, APFELgrid: a high performance tool for parton density determinations. Comput. Phys. Commun. 212, 205-209 (2017). arXiv: 1605.02070

53. D. Britzger et al., Calculations for deep inelastic scattering using fast interpolation grid techniques at NNLO in QCD and the extraction of $\alpha_{s}$ from HERA data. Eur. Phys. J. C 79(10), 845 (2019). arXiv: 1906.05303

54. A. Gehrmann-DeRidder, T. Gehrmann, N. Glover, A. Huss, T.A. Morgan, NNLO QCD corrections for $Z$ boson plus jet production. PoS RADCOR2015, 075 (2016). arXiv:1601.04569
55. S. Carrazza, Modeling NNLO jet corrections with neural networks. In: 23rd Cracow Epiphany Conference on Particle Theory Meets the First Data from LHC Run 2 Cracow, Poland, January 9-12, 2017 (2017). arXiv:1704.00471

56. J. Bellm et al., Jet cross sections at the LHC and the quest for higher precision. arXiv: 1903.12563

57. The NNPDF Collaboration, R.D. Ball et al., A determination of parton distributions with faithful uncertainty estimation. Nucl. Phys. B 809, 1-63 . arXiv:0808.1231

58. S. Alekhin et al., The PDF4LHC Working Group Interim Report. arXiv: 1101.0536

59. New Muon Collaboration, M. Arneodo et al., Accurate measurement of $F_{2}^{d} / F_{2}^{p}$ and $R_{d}-R_{p}$. Nucl. Phys. B 487, 3-26 (1997) arXiv:hep-ex/9611022

60. New Muon Collaboration, M. Arneodo et al., Measurement of the proton and deuteron structure functions, $F_{2}^{p}$ and $F_{2}^{d}$, and of the ratio $\sigma_{L} / \sigma_{T}$. Nucl. Phys. B 483, 3-43 (1997) arXiv:hep-ph/9610231

61. L.W. Whitlow, E.M. Riordan, S. Dasu, S. Rock, A. Bodek, Precise measurements of the proton and deuteron structure functions from a global analysis of the SLAC deep inelastic electron scattering cross-sections. Phys. Lett. B 282, 475-482 (1992)

62. BCDMS Collaboration, A.C. Benvenuti et al., A high statistics measurement of the proton structure functions $F_{2}\left(x, Q^{2}\right)$ and $R$ from deep inelastic muon scattering at high $Q^{2}$. Phys. Lett. B 223, 485 (1989)

63. CHORUS Collaboration, G. Onengut et al., Measurement of nucleon structure functions in neutrino scattering. Phys. Lett. B 632 65-75 (2006)

64. NuTeV Collaboration, M. Goncharov et al., Precise measurement of dimuon production cross-sections in $v_{\mu} \mathrm{Fe}$ and $\bar{v}_{\mu} \mathrm{Fe}$ deep inelastic scattering at the Tevatron. Phys. Rev. D 64112006 (2001) arXiv:hep-ex/0102049

65. D.A. Mason, Measurement of the strange-antistrange asymmetry at $\mathrm{NLO}$ in QCD from $\mathrm{NuTeV}$ dimuon data. $\mathrm{PhD}$ thesis, Oregon U. (2006)

66. ZEUS, $\mathrm{H} 1$ Collaboration, $\mathrm{H}$. Abramowicz et al., Combination of measurements of inclusive deep inelastic $e^{ \pm} p$ scattering cross sections and QCD analysis of HERA data. Eur. Phys. J. C 75(12), 580 (2015). arXiv: 1506.06042

67. H1 , ZEUS Collaboration, H. Abramowicz et al., Combination and QCD analysis of charm production cross section measurements in deep-inelastic ep scattering at HERA. Eur. Phys. J. C 73, 2311 (2013) arXiv:1211.1182

68. H1 Collaboration, F.D. Aaron et al., Measurement of the charm and beauty structure functions using the $\mathrm{H} 1$ vertex detector at HERA. Eur. Phys. J. C 65, 89-109 (2010) arXiv:0907.2643

69. Z.E.U.S. Collaboration, H. Abramowicz et al., Measurement of beauty and charm production in deep inelastic scattering at HERA and measurement of the beauty-quark mass. JHEP 09, 127 (2014). arXiv: 1405.6915

70. NuSea Collaboration, J.C. Webb et al., Absolute Drell-Yan dimuon cross sections in $800-\mathrm{GeV} / \mathrm{c} \mathrm{p} \mathrm{p} \mathrm{and} \mathrm{p} d$ collisions. arXiv:hep-ex/0302019

71. J.C. Webb, Measurement of continuum dimuon production in 800$\mathrm{GeV} / \mathrm{c}$ proton nucleon collisions. arXiv:hep-ex/0301031

72. FNAL E866/NuSea Collaboration, R.S. Towell et al., Improved measurement of the anti-d/anti-u asymmetry in the nucleon sea. Phys. Rev. D 64, 052002 (2001) arXiv:hep-ex/0103030

73. G. Moreno et al., Dimuon production in proton-copper collisions at $\sqrt{s}=38.8-G e V$. Phys. Rev. D 43, 2815-2836 (1991)

74. CDF Collaboration, T.A. Aaltonen et al., Measurement of $d \sigma / d y$ of Drell-Yan $e^{+} e^{-}$pairs in the $Z$ mass region from $p \bar{p}$ collisions at $\sqrt{s}=1.96 \mathrm{TeV}$. Phys. Lett. B 692, 232-239 (2010) arXiv:0908.3914 
75. D0 Collaboration, V.M. Abazov et al., Measurement of the shape of the boson rapidity distribution for $p \bar{p} \rightarrow Z / \gamma^{*} \rightarrow e^{+} e^{-}+X$ events produced at $\sqrt{s}=1.96-\mathrm{TeV}$. Phys. Rev. D 76, 012003 (2007) arXiv:hep-ex/0702025

76. D0 Collaboration, V.M. Abazov et al., Measurement of the muon charge asymmetry in $p \bar{p} \rightarrow \mathrm{W}+\mathrm{X} \rightarrow \mu \nu+\mathrm{X}$ events at $\sqrt{s}=1.96$ TeV. Phys. Rev. D 88, 091102 (2013) arXiv:1309.2591

77. D0 Collaboration, V.M. Abazov et al., Measurement of the electron charge asymmetry in $p \bar{p} \rightarrow W+X \rightarrow e v+X$ decays in $\boldsymbol{p} \overline{\boldsymbol{p}}$ collisions at $\sqrt{\boldsymbol{s}}=\mathbf{1 . 9 6} \mathrm{TeV}$. Phys. Rev. D 91(3), 032007 (2015) arXiv:1412.2862. [Erratum: Phys. Rev.D91,no.7,079901(2015)]

78. ATLAS Collaboration, G. Aad et al., Measurement of the highmass Drell-Yan differential cross-section in pp collisions at $\sqrt{s}=7$ $\mathrm{TeV}$ with the ATLAS detector. Phys. Lett. B 725, 223 (2013) arXiv: 1305.4192

79. ATLAS Collaboration, G. Aad et al., Measurement of the lowmass Drell-Yan differential cross section at $\sqrt{s}=7 \mathrm{TeV}$ using the ATLAS detector. JHEP 06, 112 (2014) arXiv:1404.1212

80. ATLAS Collaboration, G. Aad et al., Measurement of the inclusive $W^{ \pm}$and $Z / \gamma^{*}$ cross sections in the electron and muon decay channels in pp collisions at $\sqrt{s}=7 \mathrm{TeV}$ with the ATLAS detector. Phys. Rev. D 85, 072004 (2012) arXiv: 1109.5141

81. ATLAS Collaboration, M. Aaboud et al., Precision measurement and interpretation of inclusive $W^{+}, W^{-}$and $Z / \gamma^{*}$ production cross sections with the ATLAS detector. arXiv:1612.03016

82. ATLAS Collaboration, G. Aad et al., Measurement of the transverse momentum and $\phi_{\eta}^{*}$ distributions of Drell-Yan lepton pairs in proton-proton collisions at $\sqrt{s}=8 \mathrm{TeV}$ with the ATLAS detector. Eur. Phys. J. C 76(5), 291 (2016) arXiv:1512.02192

83. ATLAS Collaboration, G. Aad et al., Measurement of the $t \bar{t}$ production cross-section using $e \mu$ events with b-tagged jets in pp collisions at $\sqrt{s}=7$ and $8 \mathrm{TeV}$ with the ATLAS detector. Eur. Phys. J. C 74(10), 3109 (2014) arXiv:1406.5375. [Addendum: Eur. Phys. J.C76,no.11,642(2016)]

84. ATLAS Collaboration, M. Aaboud et al., Measurement of the $t \bar{t}$ production cross-section using $e \mu$ events with b-tagged jets in pp collisions at $\sqrt{s}=13 \mathrm{TeV}$ with the ATLAS detector. Phys. Lett. B 761, 136-157 arXiv:1606.02699

85. ATLAS Collaboration, G. Aad et al., Measurements of top-quark pair differential cross-sections in the lepton+jets channel in $p p$ collisions at $\sqrt{s}=8 \mathrm{TeV}$ using the ATLAS detector. Eur. Phys. J. C 76(10), 538 (2016) arXiv:1511.04716

86. C.M.S. Collaboration, S. Chatrchyan et al., Measurement of the electron charge asymmetry in inclusive $\mathrm{W}$ production in pp collisions at $\sqrt{s}=7$ TeV. Phys. Rev. Lett. 109, 111806 (2012). arXiv: 1206.2598

87. C.M.S. Collaboration, S. Chatrchyan et al., Measurement of the muon charge asymmetry in inclusive pp to WX production at $\sqrt{s}=$ $7 \mathrm{TeV}$ and an improved determination of light parton distribution functions. Phys. Rev. D 90, 032004 (2014). arXiv:1312.6283

88. CMS Collaboration, S. Chatrchyan et al., Measurement of the differential and double-differential Drell-Yan cross sections in proton-proton collisions at $\sqrt{s}=7 \mathrm{TeV}$. JHEP 1312, 030 (2013). arXiv: 1310.7291
89. CMS Collaboration, V. Khachatryan et al., Measurement of the differential cross section and charge asymmetry for inclusive $\mathrm{p} \rightarrow$ $\mathrm{W}^{ \pm}+X$ production at $\sqrt{s}=8 \mathrm{TeV}$. Eur. Phys. J. C 76(8), 469. arXiv: 1603.01803

90. CMS Collaboration, V. Khachatryan et al., Measurement of the $Z$ boson differential cross section in transverse momentum and rapidity in proton-proton collisions at $8 \mathrm{TeV}$. Phys. Lett. B 749, 187-209 arXiv:1504.03511

91. CMS Collaboration, V. Khachatryan et al., Measurement of the $\mathrm{t}$-tbar production cross section in the e-mu channel in protonproton collisions at sqrt(s) $=7$ and $8 \mathrm{TeV}$. JHEP 08, 029 (2016). arXiv: 1603.02303

92. CMS Collaboration, V. Khachatryan et al., Measurement of the top quark pair production cross section in proton-proton collisions at $\sqrt{(} s)=13 \mathrm{TeV}$. Phys. Rev. Lett. 116(5), 052002 (2016) arXiv: 1510.05302

93. CMS Collaboration, V. Khachatryan et al., Measurement of the differential cross section for top quark pair production in $\mathrm{pp}$ collisions at $\sqrt{s}=8 \mathrm{TeV}$. Eur. Phys. J. C 75(11), 542 (2015) arXiv: 1505.04480

94. LHCb Collaboration, R. Aaij et al., Inclusive $W$ and $Z$ production in the forward region at $\sqrt{s}=7 \mathrm{TeV}$. JHEP 1206, 058 (2012) arXiv: 1204.1620

95. LHCb Collaboration, R. Aaij et al., Measurement of the crosssection for $Z \rightarrow e^{+} e^{-}$production in $p p$ collisions at $\sqrt{s}=7$ TeV. JHEP 1302, 106 (2013) arXiv:1212.4620

96. LHCb Collaboration, R. Aaij et al., Measurement of the forward $Z$ boson production cross-section in $p p$ collisions at $\sqrt{s}=7 \mathrm{TeV}$. JHEP 08, 039 (2015). arXiv: 1505.07024

97. LHCb Collaboration, R. Aaij et al., Measurement of forward W and $\mathrm{Z}$ boson production in $p p$ collisions at $\sqrt{s}=8 \mathrm{TeV}$. JHEP 01, 155 (2016). arXiv:1511.08039

98. The NNPDF Collaboration, R.D. Ball et al., Fitting parton distribution data with multiplicative normalization uncertainties. JHEP 05, 075 (2010) arXiv:0912.2276

99. Z. Kassabov, Reportengine: a framework for declarative data analysis. https://doi.org/10.5281/zenodo.2571601 (2019)

100. R.D. Ball, S. Carrazza, L. Del Debbio, S. Forte, J. Gao et al., Parton distribution benchmarking with LHC data. JHEP 1304, 125 (2013). arXiv: 1211.5142

101. NNPDF Collaboration, R.D. Ball et al., Parton distributions for the LHC Run II, JHEP 04, 040 (2015) arXiv: 1410.8849

102. R. Abdul Khalek, S. Bailey, J. Gao, L. Harland-Lang, J. Rojo, Towards ultimate parton distributions at the high-luminosity LHC. Eur. Phys. J. C 78(11), 962 (2018). arXiv:1810.03639

103. S. Marzani, G. Soyez, M. Spannowsky, Looking Inside Jets: An Introduction to Jet Substructure and Boosted-Object Phenomenology, vol. 958 (Springer, Berlin, 2019) 